\title{
Functional Heterogeneity in Neighboring Neurons of Cat Primary Visual Cortex in Response to Both Artificial and Natural Stimuli
}

\author{
Kevan A.C. Martin and Sylvia Schröder \\ Institute of Neuroinformatics, University of Zürich and Eidgenössische Technische Hochschule Zürich, 8057 Zürich, Switzerland
}

Neurons in primary visual cortex of many mammals are clustered according to their preference to stimulus parameters such as orientation and spatial frequency. Nevertheless, responses to complex visual stimuli are highly heterogeneous between adjacent neurons. To investigate the relation between these observations, we recorded from pairs of neighboring neurons in area 17 of anesthetized cats in response to stimuli of differing complexity: sinusoidal drifting gratings, binary dense noise, and natural movies. Comparisons of the tuning curves revealed similar orientation and direction preferences for neighboring neurons, but large differences in preferred phase, direction selectivity, and tuning width of spatial frequency. No pair was similar across all tuning properties. The neurons' firing rates averaged across multiple stimulus repetitions (the "signal") were also compared. Binned between 10 and $200 \mathrm{~ms}$, the correlation between these signals was close to zero in the median across all pairs for all stimulus classes. Signal correlations agreed poorly with differences in tuning properties, except for receptive field offset and relative modulation (i.e., the strength of phase modulation). Nonetheless, signal correlations for different stimulus classes were well correlated with each other, even for gratings and movies. Conversely, trial-to-trial fluctuations (termed "noise") were poorly correlated between neighboring neurons, suggesting low degrees of common input. In response to gratings and visual noise, signal and noise correlations were well correlated with each other, but less so for responses to movies. These findings have relevance for our understanding of the processing of natural stimuli in a functionally heterogeneous cortical network.

\section{Introduction}

Since the seminal findings of Mountcastle (1957) and Hubel and Wiesel $(1962,1968)$, we know that sensory cortex of cats and monkeys is organized into columns of neurons with shared functional preferences. This columnar architecture seems to offer at least two advantages: minimized wiring — all circuits that need to deal with one patch of sensory surface are colocalized (Hubel and Wiesel, 1963; Chklovskii and Koulakov, 2004)—and a means of averaging across intrinsic neuronal noise (Shadlen et al., 1996; Parker and Newsome, 1998; Mazurek and Shadlen, 2002). In contrast, theoretical considerations on energy and coding efficiency argue for sparse coding in which only a few cells are active at any moment in time (Olshausen and Field, 2004).

To understand information coding in visual cortex, it seems relevant to investigate functional differences between neurons at

Received Aug. 24, 2012; revised March 4, 2013; accepted March 12, 2013.

Author contributions: K.A.C.M. and S.S. designed research; K.A.C.M. and S.S. performed research; S.S. contributed unpublished reagents/analytic tools; S.S. analyzed data; K.A.C.M. and S.S. wrote the paper.

This work was supported by EU Daisy FP6 015803, EU SECO FP7 216593, and by ETH Grant 2-73246-08 to K.A.C.M. We thank Nuno M. da Costa and Elisha Ruesch for their help in performing the experiments and Sepp Kollmorgen for many discussions on data analysis. We also thank Simone Rickauer for performing most of the histological processing as well as John Anderson, Andreas Keller, and Isabelle Spühler for their support during the experiments. Furthermore, we thank the reviewers for their detailed and valuable critique and comments.

The authors declare no competing financial interests.

Corresponding author: Sylvia Schröder, Institute of Neuroinformatics, University of Zürich and ETH Zürich, Winterthurerstrasse 190,8057 Zürich, Switzerland. E-mail: sschroed@ini.phys.ethz.ch.

DOI:10.1523/JNEUROSCI.4071-12.2013

Copyright $\odot 2013$ the authors $\quad 0270-6474 / 13 / 337325-20 \$ 15.00 / 0$ a very fine spatial scale. We know that, compared with distant ones, neighboring neurons are more likely to receive similar synaptic input and to project to similar targets. Few studies, however, have compared the responses and receptive fields (RFs) of neighboring neurons. DeAngelis et al. (1999) investigated spatiotemporal RFs and found several parameters that are as different between nearby neurons as between distant neurons. However, their analyses were confined to simple cells and it is difficult to infer from their results how differently nearby neurons would respond to natural stimuli, to which cortical coding strategies are probably adapted (Field, 1987). From knowledge of the RF structure, current models predict poorly a complex cell's response to natural stimuli (Carandini et al., 2005). Furthermore, RF structure changes somewhat with stimulus class (David et al., 2004). Studies that used more complex stimuli, such as binary dense noise, Walsh patterns, or natural images and movies, found very small signal correlations (i.e., the correlation between firing rates averaged across multiple stimulus repetitions), reflecting large response heterogeneities between neighboring neurons (Gawne et al., 1996; Reich et al., 2001; Weliky et al., 2003; Yen et al., 2007). These studies, however, rarely differentiate between different time scales of responses (although the relevant scale for coding is unknown) and do not relate their results to differences between the neurons' RF properties.

Of further significance for population coding are the correlations between the neurons' trial-to-trial fluctuations in response to the same stimulus, which is called "noise" and is thought to 
reflect their common input. The strength of these noise correlations can alter coding capacities tremendously (Abbott and Dayan, 1999; Averbeck et al., 2006). Previous studies in a variety of brain areas found small, positive noise correlations (for review, see Cohen and Kohn, 2011), but rarely considered changes across stimulus classes or how they relate to signal correlations measured at time scales shorter than hundreds of milliseconds.

In this study, we quantified differences between RFs, signal correlations, and noise correlations of neighboring neurons in cat primary visual cortex in response to three different stimulus classes. We then related these three measures to each other using varying time scales and compared the results across stimulus classes and across cortical layers.

\section{Materials and Methods}

\section{Animal preparation}

All experiments, animal treatment and surgical protocols were carried out with authorization and under a license granted to K.A.C.M. by the Cantonal Veterinary Office of Zürich, Switzerland. The data presented here originate from 15 adult cats $(2.2-4.3 \mathrm{~kg})$ of either sex. The animals were initially anesthetized with a subcutaneous injection of xylazine ( $\sim 0.5 \mathrm{mg} / \mathrm{kg}$; Rompun $2 \%$; Bayer) and ketamine ( $\sim 15 \mathrm{mg} / \mathrm{kg}$; Narketan 10 ; Vétoquinol). The femoral vein and artery and the trachea were cannulated while the cat was maintained under general anesthesia with $\sim 2 \%$ halothane (Arovet) in oxygen/nitrous oxide (50\%/50\%) and with regular intravenous injections of alphaxalone/alfadolone (Saffan, ScheringPlough Animal Health). Throughout the experiment, alphaxalone/alfadolone ( $\sim 5-14$ and 2-5 mg/kg/h, respectively; Saffan, Schering-Plough Animal Health) was continuously delivered intravenously to maintain general anesthesia. The cat was artificially ventilated with oxygen/nitrous oxide $(30 \% / 70 \%)$ and the ventilation volume was adjusted so that endtidal $\mathrm{CO}_{2}$ remained at a level of $\sim 4.5 \%$. After opening the skull, the cat was given an intravenous injection of the muscle relaxant gallamine triethiodide (40 mg; Sigma-Aldrich) and thereafter gallamine triethiodide $(\sim 7.3 \mathrm{mg} / \mathrm{kg} / \mathrm{h}$; Sigma-Aldrich) mixed with $(+)$-tubocurarine chloride hydrate $(\sim 0.7 \mathrm{mg} / \mathrm{kg} / \mathrm{h}$; Sigma-Aldrich) was delivered intravenously to prevent eye movements. Lidocain gel (4\%; G. Streuli) was applied to all pressure points (ear bars and rectal thermometer). Topical antibiotics (Voltamicin; OmniVision) and atropine (1\%; Ursapharm) to prevent accommodation were applied to the eyes before they were covered with gas-permeable, neutral power contact lenses. The nictitating membranes were retracted with phenylephrine (5\%; Bausch \& Lomb). During the course of the experiment, electroencephalogram (EEG, maintained in spindling state) and electrocardiogram readings and blood pressure (measured via cannula in femoral artery) were monitored continuously. If needed, additional intravenous Saffan injections or halothane $(0-2 \%$; Arovet) could be given. A thermistor-controlled heating blanket, on which the cat was lying, kept the cat's rectal temperature at $37^{\circ} \mathrm{C}$.

The location of the blind spot of each eye was marked on the screen used for mapping the RFs. This allowed the position of the area centralis to be estimated for each eye. Appropriate spectacle lenses were used to focus the eyes onto the screen positioned $114 \mathrm{~cm}$ in front of the eyes. A small craniotomy was performed over area 17 (Horsley-Clark coordinates anteroposterior -3 to -6 and mediolateral 0 to 3 ). A recording chamber was mounted over the craniotomy and a tiny durotomy was made at the recording site. After the electrode was lowered to the surface of the brain, the chamber was filled with agar (Sigma-Aldrich) for stabilization.

\section{Electrophysiology and extracellular labeling}

To record the neural signals, a glass micropipette with tip diameter of 2-4 $\mu \mathrm{m}$ (for 4 recordings, the tip was beveled) filled with $1 \mathrm{~mol} / \mathrm{l}$ potassium acetate (in a few cases with $0.05 \mathrm{~mol} / \mathrm{l}$ Tris and $0.2 \mathrm{~mol} / \mathrm{l} \mathrm{KCl}$ with $2 \%$ horseradish peroxidase) and with a chlorided silver wire electrode was used. The pipettes had an average resistance of $25 \mathrm{M} \Omega$ (range, 6-90). The reference electrode (chlorided silver wire) for the recordings was attached to the scalp a few centimeters from the recording chamber. A second, low-impedance glass pipette was used to record the local field potential (data not used in this study). It was glued to the highimpedance pipette at an average tip-to-tip distance of $34 \mu \mathrm{m}$ (range, $16-60)$ and the second pipette was used to mark the recording site. For this purpose, the second pipette was filled with a solution of $2 \%$ Pontamine Sky Blue (6B; Sigma-Aldrich) in $0.5 \mathrm{~mol} / 1 \mathrm{NaCl}, 0.5 \mathrm{~mol} / \mathrm{l}$ potassium acetate, and $0.01 \mathrm{~mol} / \mathrm{l}$ phosphate buffer. After successful recordings, this solution was injected iontophoretically into the extracellular space with current pulses of $3 \mathrm{~s}$ on $/ 3 \mathrm{~s}$ off, amplitude of $4-5 \mu \mathrm{A}$, and duration of $2-5 \mathrm{~min}$. The injection left a blue spot in the tissue so that the cortical layer of the recording could be determined. The electrical signals from the high-impedance pipette were recorded with an Axoprobe-1A system (Axon Instruments), further amplified, and filtered in a band of $100-8000 \mathrm{~Hz}$ (NeuroLog and Kemo), and then digitized at $20 \mathrm{kHz}$ with a 12-bit resolution (CED 1401 and Spike2 software; CED).

\section{Perfusion and histology}

At the end of an experiment, the cat was given an overdose of intravenous anesthetic sufficient to flatten the EEG. The cat was then perfused transcardially with normal $0.9 \% \mathrm{NaCl}$ solution followed by a solution of $4 \%$ paraformaldehyde, $0.3 \%$ gluteraldehyde, and $15 \%$ saturated solution of picric acid in $0.1 \mathrm{~mol} / 1$ phosphate buffer. After fixation, the brain was stereotaxically cut and the block containing the recording sites was removed from the skull. Each block of brain tissue was Vibratome sectioned at $80 \mu \mathrm{m}$ in the coronal plane. After the slices were flat mounted onto glass slides, cell bodies were made visible with a Nissl or neutral red stain so that cortical layers could be distinguished.

\section{Visual stimuli}

Before each recording, the RFs of one or more cells were plotted by hand and the location, size, ocular dominance, orientation and direction preference, and RF type (simple or complex) were determined. The centers of the RFs had an average distance of approximately $4^{\circ}$ from the estimated area centralis (always $<10^{\circ}$ ) and an average size of $1.25^{\circ}$ (along preferred orientation $) \times 1.2^{\circ}$ (orthogonal to preferred orientation). Computergenerated stimuli were presented on a Sony CPD-G500 monitor under control of a ViSaGe graphics card (Cambridge Research Systems). The monitor placed at $114 \mathrm{~cm}$ in front of the cat's eyes had an image area of $19.1^{\circ} \times 14.4^{\circ}$ at a resolution of $800 \times 600$ pixels. The frame rate was 100 Hz. All stimuli were centered approximately on the center of the manually measured RF, extended well beyond the classical RFs of the recorded neurons, and were presented monocularly to the dominant eye (except for two cases in which only binocular stimulation was effective).

Sinusoidal drifting gratings were presented in a square aperture with edge lengths of $4-6^{\circ}$ on a mean gray background. Gratings were first varied in orientation and direction (in most cases), then in spatial frequency, and then in temporal frequency using appropriate step sizes and ranges depending on the selectivity of the neurons. Orientation was varied in steps of $3.75-22.5^{\circ}$ (median, $18^{\circ}$ ), spatial frequency in steps of $0.08-0.25$ cycles per degree (median, 0.2 ), and temporal frequency in steps of $0.1-0.4$ cycles/s (median, 0.25 ). After the tuning to a given parameter was established, the parameter was fixed to a value close to the preferences of all simultaneously recorded neurons. In a few cases, the parameter was fixed to each neuron's optimum separately to measure the tuning to the other stimulus parameters. To find the optima for a given neuron, tuning curves were estimated online using online spike sorting (Spike2, Cambridge Electronic Design). Gratings had low contrasts of $10-50 \%$ to prevent response saturation. They were shown for $5 \mathrm{~s}$ (in 3 recordings for $3 \mathrm{~s}$ ) and were interleaved for $2 \mathrm{~s}$ (in 1 case for $10 \mathrm{~s}$ ) with a blank mean gray screen. Gratings were presented in random order and in most cases each one was repeated 10 times (at least five times) except when the recording had to be stopped prematurely due to loss of cells.

The natural movie scenes are digitized broadcasts from Dutch, British, and German television taken from Hans van Hateren's image and movie database (van Hateren and Ruderman, 1998). The movie images have a resolution of $128 \times 128$ pixels. To get an image size of $6.17^{\circ} \times 6.17^{\circ}$, each frame was magnified to $256 \times 256$ pixels by quadruplicating each pixel. Gray scale values of each movie were discretized to 255 values and were scaled so that the brightest and darkest pixels reached maximal and minimal luminance, respectively. The movies were placed on a mean gray 
background and presented at $50 \mathrm{~Hz}$ (the original frame rate of the movies in the database) or at $25 \mathrm{~Hz}$ (after averaging pairs of consecutive frames). Each movie clip lasted for $10 \mathrm{~s}$, contained no video cuts, and was repeated 30 times (in four pair recordings, movies were repeated only 10-20 times). The clips were interleaved by $3.7-4.5 \mathrm{~s}$ of a blank mean gray screen. For neurons that were lost before the presentation was completed, only data for movies (or parts thereof) that were presented for at least five trials were considered in further analyses.

The third stimulus type was binary dense noise consisting of elongated bars at an orientation intermediate between the preferred orientations of the neurons. The bars occurred in a grid with $1-4$ rows and 5-15 columns. The grid had a width of $2.6-6^{\circ}$ and a height of $2.4-5.3^{\circ}$ and appeared on a mean gray background. Each image was presented for 20 $\mathrm{ms}$ (i.e., for two video frames). The sequence of images was produced by changing the luminance value of each bar between white and black according to a pseudorandom binary m-sequence of order 12 (Pinter and Nabet, 1992). The same sequence was used for each bar, but was temporally shifted so that luminance values of all bars were uncorrelated with each other (shifts were determined as ratio between the total length of the $\mathrm{m}$-sequence in frames and the total number of bars resulting in 91-455 frames). The same image sequence was repeated 10 times without breaks between repetitions. In total, the complete presentation of the noise stimulus lasted for $\sim 13 \mathrm{~min}$ and $39 \mathrm{~s}$.

\section{Data analysis}

Spike sorting. Continuous voltage traces were recorded and action potentials were detected and sorted using the offline sorting algorithm WaveClus (Quiroga et al., 2004), which is a publicly available MATLAB (MathWorks) toolbox. Before spike detection, the raw voltage traces were band-pass filtered between 200 and $3000 \mathrm{~Hz}$ with an elliptic filter in forward and reverse directions to prevent phase distortions. The spikes clustered by WaveClus were visually checked for possible false assignments. Spikes that were not assigned to any cluster or that were manually discarded were screened for possibly overlapping spikes originating from two neurons. Overlapping wave forms were decomposed by matching them with templates of the previously found spike clusters (Atiya, 1992). Matches were manually inspected and corrected if necessary. This procedure recovered spike occurrences that would go undetected with spikesorting algorithms only based on the similarity between waveforms. Examples of sorted spikes and resolved overlapping spikes are shown in Figure 1 .

Tuning curves and phase analysis. One approach to quantifying response differences between neighboring neurons is based on a comparison of their tuning curves. The curves are described by various functions fitted to the median responses of a neuron to sinusoidal drifting gratings that varied in one parameter while all other parameters were fixed. The magnitude of a neuron's response during one trial was determined depending on the RF type (Cavanaugh et al., 2002). For "simple" RFs (criteria described below), spikes were aligned to the onset of each grating cycle, a Fourier transformation was applied, and the response, termed "F1", was defined as half of the peak-to-peak amplitude of the first harmonic. For "complex" RFs, the response in one trial, termed "DC," was determined as the mean spike rate during grating presentation minus the baseline firing rate (i.e., the mean spike rate during presentation of the blank screen immediately before and after the trial; the first $250 \mathrm{~ms}$ of these responses were not considered to discard any off-responses). The RF was classified as "simple" if the spatial frequency curve based on F1 responses had a larger maximum than the curve based on DC responses, otherwise the RF was classified as "complex" (Cavanaugh et al., 2002). If no responses to varying spatial frequency were recorded, the RF type was inferred from the neuron's orientation tuning curve in the same way. The ratio between the maxima estimated from $\mathrm{F} 1$ and DC responses is termed "relative modulation" and will be used for comparison of tuning properties between neighboring neurons.

For each of the stimulus parameters, orientation/direction, spatial frequency, and temporal frequency, several functions were fit to the median responses of a neuron by minimizing the reduced $\chi^{2}$ error between the observed and the estimated responses. The methods of Cavanaugh et al. (2002) were used and the reduced $\chi^{2}$ error was defined as follows:

$$
\chi^{2}=\sum_{i=1}^{n} \frac{\left(e_{i}-o_{i}\right)}{\sigma_{i}^{2}} / d f
$$

where $n$ is the number of different values of the stimulus parameter, $e_{\mathrm{i}}$ is the estimated response to the $i^{\text {th }}$ value, $o_{\mathrm{i}}$ is the observed median response across all repetitions (at least 3 per stimulus), $\sigma_{i}^{2}$ is the squared mean absolute deviation from the mean, and $d f$ are the degrees of freedom of the function used to fit the responses. To avoid very large values of $\chi^{2}, \sigma_{i}^{2}$ was set to be at least $0.01 \rho \cdot \max o_{i}$, with $\rho=\left\langle\sigma_{i}^{2} / o_{i}\right\rangle_{i}$, where $\langle\cdot\rangle_{i}$ is the mean across i. $\rho$ is analogous to the variance-to-mean ratio.

For each tuning property, the responses were fitted to at least one function that was chosen for each stimulus parameter separately according to the expected shape of the tuning curve. To control for the situation in which responses were not tuned, the responses were also fit to a horizontal line at variable height. If the fit to the horizontal line resulted in the smaller reduced $\chi^{2}$ error, responses were not considered in the comparison between tuning properties. In all other cases, the best-fitting function was used to extract the relevant tuning properties, which are the neuron's preferred feature value, tuning width, and the direction index for direction tuning. Responses to gratings varying in orientation and direction of movement were fit to a wrapped Gaussian, $G(\theta)=A \Sigma_{n=-\infty}^{\infty} \exp \left\{-(\theta-\mu+180 n)^{2} /\left(2 \sigma^{2}\right)\right\}$, with two peaks separated by $180^{\circ}$ (Swindale, 1998) if gratings of opposite directions of movements were shown (Fig. $3 A$ shows examples of three neurons) or to a simple Gaussian if only gratings of directions within $180^{\circ}$ were presented. The cell's preferred orientation was defined as the value of the Gaussian's peak modulo $180^{\circ}$, whereas preferred direction was defined as the value of the highest peak only if gratings of opposite directions were presented. The width of orientation tuning was determined from the width of the larger Gaussian at half-height (i.e., halfway between zero and the curve's maximum). The direction selectivity of the cell was estimated by the direction index defined as DI $=\left(R_{p}-R_{o}\right) /\left(R_{p}+R_{o}\right)$ (as in Reid et al., 1987), where $R_{p}$ is the response to the preferred direction, $R_{o}$ the response to the opposite direction. For varying spatial frequencies, responses were fit to a double half-Gaussian and to a logarithmic double half-Gaussian (Baker et al., 1998; Fig. 3B shows examples of three neurons). Responses to gratings varying in temporal frequency were fit to a Gaussian and to a logarithmic Gaussian (Nover et al., 2005; Fig. 3C shows examples). Preferred spatial and temporal frequencies were taken as the values at the Gaussian's peak, whereas the tuning width for both parameters was defined as the width at half-height in terms of octaves. For all stimulus features, the tuning width of a neuron was only considered if the lower and upper value at half-width was inside or very close to the range of sampled values. Therefore, eight neurons were excluded from measurements of orientation tuning width and eight neurons from measurements of spatial frequency tuning width (seven of these neurons were lowpass-filtering cells). Unfortunately, in the case of temporal frequency, values high enough to capture the complete tuning range of the cells could not be sampled. To prevent false estimates, the neuron's preferred temporal frequency was only considered if it was estimated to be smaller than at least the three largest sampled values. The largest preferences of temporal frequency included were approximately 2.5 cycles/s (see "Tuning Differences" for how this affected the results on differences between neighboring neurons). For the same reason, temporal frequency tuning width was excluded from the comparison between neighboring neurons.

To fit the functions to the cell's responses, an unconstrained nonlinear optimization procedure (function fminunc, MATLAB; MathWorks) was used in case of the straight horizontal line or a constrained nonlinear optimization procedure for the various other functions (function fmincon, MATLAB; MathWorks). Constraints for the latter fitting procedure were introduced to prevent unreasonable fits and included restrictions on the position of the peak of the Gaussian and restrictions on its width. The goodness-of-fit of the curves was assessed as follows: 
A

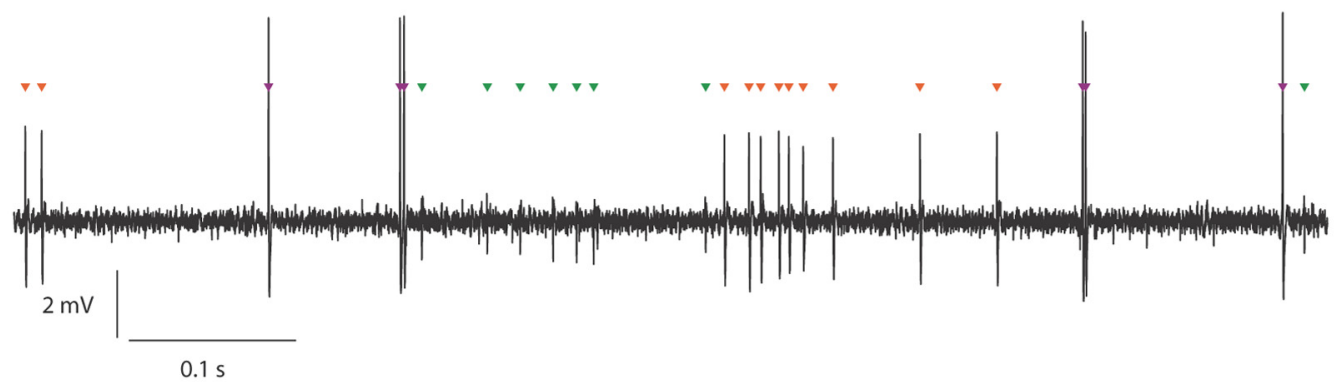

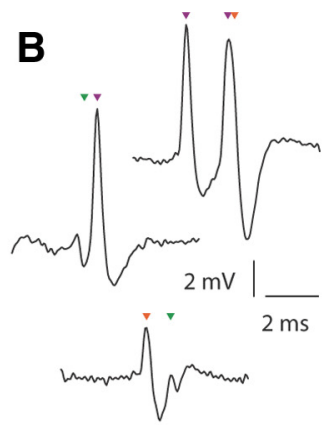

\section{C}
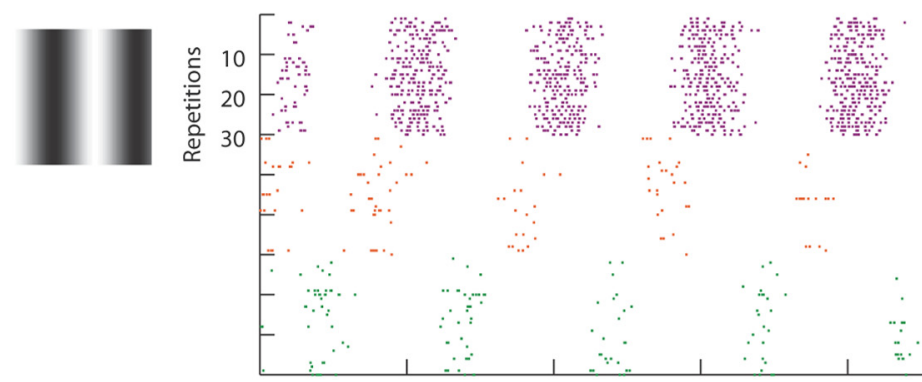

Grating
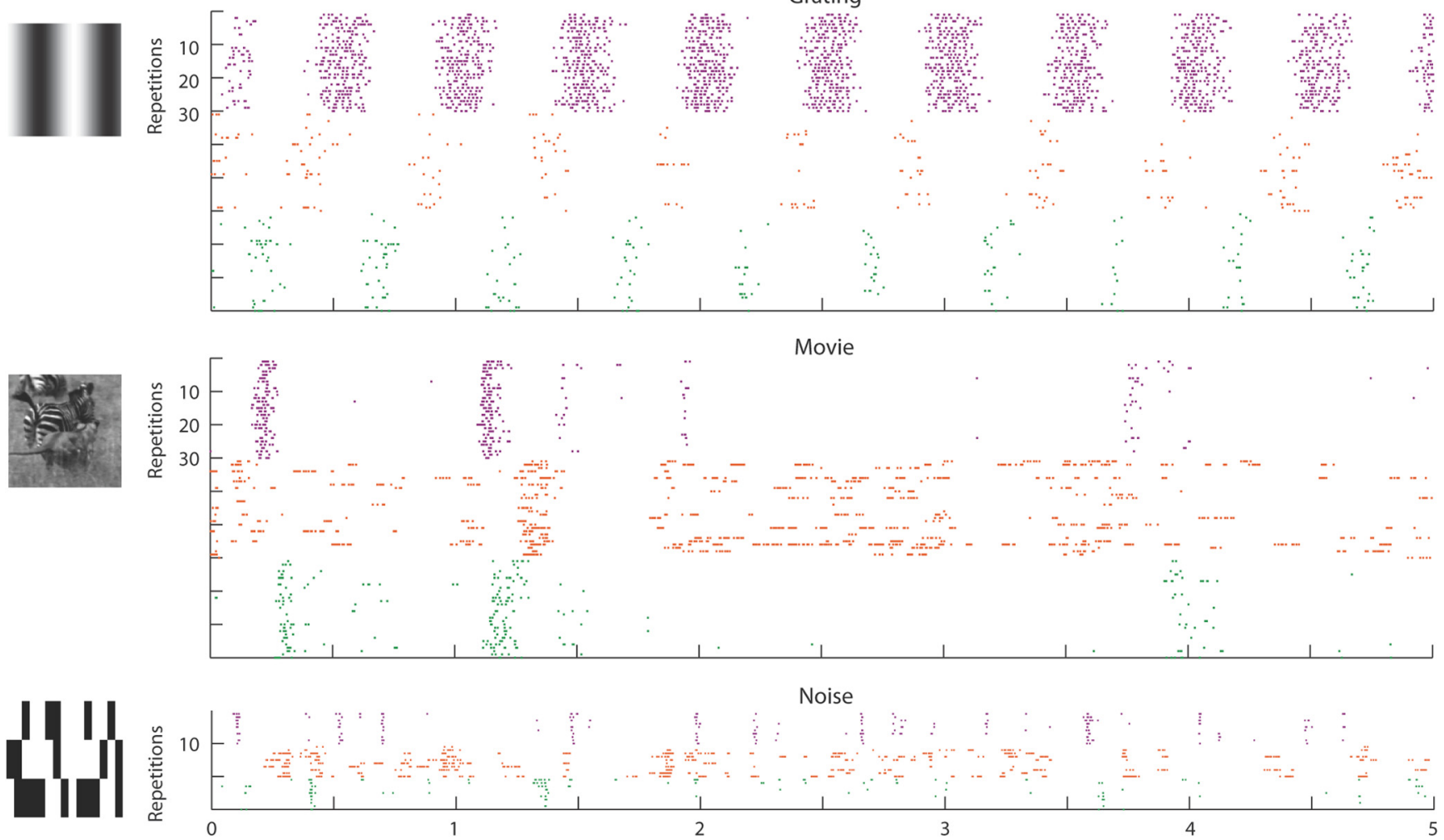

Noise 
ences between random neurons, a permutation test previously introduced by DeAngelis et al. (1999) was used. Neurons that were recorded at different sites were picked randomly and their absolute difference in the respective stimulus parameter was measured. The number of random pairs selected matched the number of original pairs. In this way, 1000 random distributions were generated for each stimulus feature and the medians of these distributions (termed random medians) were used for comparison with the median of the original data. Differences between neighboring neurons were counted as significantly smaller than expected if the median of the original differences was smaller than at least $95 \%$ of the random medians. The degree of clustering of a tuning parameter was assessed with a measure called the "clustering index," which was also devised in the analyses of DeAngelis et al. (1999). It is the ratio between the median difference among neighboring neurons and the median of all random medians (see above) for one tuning parameter. If the clustering index is 1 , neighboring neurons are as similar in the parameter as random neurons are; the more it exceeds 1 , the stronger is the clustering in cortex.

Reconstruction of RFs from responses to visual noise. A further tuning parameter measured was the position of the RF, which was reconstructed from the neurons' responses to the visual noise stimuli (recorded in 12 pairs and one triplet). First, the spike-triggered average (STA) was determined and then the eigenvector of the spike-triggered covariance matrix with the largest eigenvalue, as well as their significance, was measured following the procedure outlined by Schwartz et al. (2006). For both quantities, the last 10 frames (equivalent to $200 \mathrm{~ms}$ ) that occurred before each spike were considered. Although this time range does not always cover the complete spatiotemporal RF of a neuron, it always includes its maximum, which is most important for the estimation of RF center and spatial extension. In short, for calculating the STA and the first eigenvector, the mean was first subtracted from the stimulus ensemble, which was then whitened. The STA consists of the stimulus frames occurring directly before the spikes averaged across all spike occurrences. The first eigenvector of the covariance matrix of the spike-triggered stimulus ensemble was determined after the STA was projected out of the stimulus ensemble. To test the significance of both measures, a distribution of random STAs and eigenvectors was generated by bootstrapping, which involved shifting the spikes by a random amount relative to the stimulus sequence. STA and eigenvector were considered significant if they exceeded $97.5 \%$ of the random distribution. Estimation of the center of the $\mathrm{RF}$ was based on a two-dimensional (one time and one space dimension) variant of each significant filter (STA or first eigenvector). For this, only one row of bars (orthogonal to their orientation) of each filter was considered (the central row or the one with the largest amplitude). Each frame of these spatiotemporal filters was convolved with a Gaussian (with an SD of 0.65 pixels and a radius of 2.5 pixels). The filter was then fit to an RF model constructed as the weighted sum of two space-time separable components as described by DeAngelis et al. (1999). Each component was modeled as the product of a spatial waveform, $G(X)$, and a temporal waveform, $H(T)$, as follows:

$$
R(X, T)=K\left[G_{1}(X) H_{1}(T)+\alpha G_{2}(X) H_{2}(T)\right]
$$

where $K$ is an overall scaling factor, and $\alpha$ is a weight on the second separable subunit. $G(X)$ is a Gabor function:

$$
G_{1 ; 2}(X)=\exp \left(-\left(\frac{2\left(X-X_{0}\right)}{w}\right)^{2}\right) \cos \left(2 \pi f\left(X-X_{0}\right)+P_{1 ; 2}\right)
$$

where $X_{0}, w, f$, and $P_{1 ; 2}$ are free parameters: $X_{0}$ and $w$ represent the center and width of the Gaussian RF envelope, $f$ and $P_{1 ; 2}$ correspond to the spatial frequency and phases of the sinusoids. $G_{2}(X)$ differs from $G_{1}(X)$ only by a shift in its phase by $90^{\circ}\left(P_{2}=P_{1} \pm 90^{\circ}\right) . H_{1 ; 2}(T)$ has the same form as $G_{1 ; 2}(X)$, but describes the temporal dimension and is temporally skewed by replacing $X$ with $T_{\mathrm{s}}=2 \arctan (\beta T) / \pi$. $X_{0}$ now represents the peak latency response, $w$ the response duration, $f$ the temporal frequency, and $P_{1 ; 2}$ the temporal phases. Again, $H_{2}(T)$ is phase shifted by $90^{\circ}$ relative to $H_{1}(T)$. See DeAngelis et al. (1999) for a more detailed description of the model and for depictions of fits to real data. The fits were evaluated by their fitting error, $\Sigma_{X, T}\left(R F_{X, T}-R(X, T)\right)^{2} / \Sigma_{X, T} R F_{X, T}^{2}$, where $R F_{X, T}$ is the
STA or the first eigenvector. Only fits resulting in errors smaller than 0.45 were considered. The center of the RF was then defined by the spatial parameter $X_{0}$ of the fit that resulted in the smaller error.

Correlation measures. The second approach for comparing stimulusdependent responses of neighboring neurons was to measure their signal correlations at various time scales. Signal correlations that were measured on complete trials of grating stimuli are termed "trial correlations." They are defined as Pearson's correlation between average firing rates of two neurons in response to all presented grating stimuli as follows:

$$
\text { rho }=\frac{\sum_{i=1}^{n}\left(X_{i}-\bar{X}\right)\left(Y_{i}-\bar{Y}\right)}{\sqrt{\sum_{i=1}^{n}\left(X_{i}-\bar{X}\right)^{2}} \sqrt{\sum_{i=1}^{n}\left(Y_{i}-\bar{Y}\right)^{2}}}
$$

where $X_{\mathrm{i}}$ is the mean firing rate of one neuron for the complete duration of grating stimulus $i$ averaged across all repetitions, $\bar{X}$ is the mean across all $X_{\mathrm{i}}, Y_{\mathrm{i}}$ and $\bar{Y}$ are the analogous data of the second neuron, and $n$ is the total number of stimuli. The set of firing rates was normalized for each stimulus parameter (e.g., orientation) separately before combining across all parameters (Fig. 6A). Specifically, a $z$-transformation was applied (i.e., by subtracting the mean and dividing by $1 \mathrm{SD}$ ) to all responses given to stimuli varying in orientation before combining them with the $z$-transformed responses to stimuli varying in spatial frequency and temporal frequency, respectively. Only then was the trial correlation calculated. This was necessary to compensate for differences in responsiveness due to different sets of fixed parameters, which could have led to spurious correlations (fixing orientation to the preferred one might lead to generally higher firing rates than fixing another parameter while orientation is varied). Because a good estimate of signal correlations depends on an accurate estimate of the neurons' firing rates, only reliable responses were taken into account. To test for reliability, responses $\left(X_{i}^{j}, X_{i}^{k}\right)$ were paired to the same stimulus $i$ but from different trials $j$ and $k$, and paired responses from all stimuli that were varied in one parameter were pooled together. If the two vectors $X_{i}^{j}$ and $X_{i}^{k}$ (for all $i=1, \ldots, n$ and all $j=1, \ldots, T-1$, $k=2, \ldots, T, j<k$, where $T$ is the number of trials) were significantly correlated ( $p<0.05$, permutation test, see below for description), the neuron's responses to this stimulus parameter were said to be reliable and were considered for trial correlation. Furthermore, the trial correlation of a pair was only taken into account if responses to at least 10 stimuli were measured and reliable for both neurons. Each stimulus was repeated at least five times.

Signal correlations at shorter time scales of $10-200 \mathrm{~ms}$ are defined in a similar way as above:

$$
\text { rho }=\frac{\sum_{i=1}^{n} \sum_{b=1}^{L}\left(X_{i, b}-\bar{X}\right)\left(Y_{i, b}-\bar{Y}\right)}{\sqrt{\sum_{i=1}^{n} \sum_{b=1}^{L}\left(X_{i, b}-\bar{X}\right)^{2}} \sqrt{\sum_{i=1}^{n} \sum_{b=1}^{L}\left(Y_{i, b}-\bar{Y}\right)^{2}}}
$$

where $X_{i, b}$ and $Y_{i, b}$ represent firing rates in bin $b$ averaged across all repetitions of stimulus $i, \bar{X}$ and $\bar{Y}$ are the averages across all stimuli and all bins, and $L$ is the number of bins. For each stimulus class, responses to all stimuli of that class were considered in calculating a pair's signal correlation. As was done for trial correlations, binned responses to gratings varying in one stimulus parameter were $z$-transformed separately before signal correlation was determined. All responses to stimuli that did not elicit reliable responses were discarded. Again, vectors $X_{i, b}^{j}$ and $X_{i, b}^{k}$ were constructed for all $b=1, \ldots, L$, and for all trials $j=1, \ldots$, $T-1, k=2, \ldots, T, j<k$. The responses to the stimulus were considered reliable if the vectors were significantly correlated with each other. Signal correlations were considered only if responses to at least 10 bins were measured and if stimuli were repeated at least five times.

Noise correlations compare the neurons' response deviations from their mean response and are commonly attributed to shared input between the neurons. Noise correlations were determined in a similar way as shown in formulas (1) and (2) above. In formula (1), $X_{i}$ was replaced by $\left(X_{i}^{j}-X_{i}\right) / \sigma_{i}$, where $X_{i}^{j}$ is the mean firing rate for the complete duration of grating stimulus $i$ in trial $j$, and $\sigma_{i}$ is the SD across all trials. 
Similarly, in formula (2), $X_{i, b}$ was replaced by $\left(X_{i, b}^{j}-X_{i, b}\right) / \sigma_{i, b}$, where $X_{i, b}$ is the firing rate in bin $b$ of stimulus $i$ averaged across all trials, and $\sigma_{i, b}$ is the SD of responses in bin $b$ of stimulus $i$. In both formulas, the analogous replacements are done for responses of the second neuron, and a sum across trials is added in the nominator and denominator. Similar to signal correlations, noise correlations were measured at complete trials of grating stimuli and at bins of 10-200 ms for all stimulus classes and only reliable responses were considered (see above). All stimuli that belong to one class and that were repeated at least five times were used to calculate each pair's noise correlation.

Significance test for correlation measures. A permutation test was performed to determine the significance of the strength of a correlation between vectors $X$ and $Y$. The entries in $Y$ were randomly permuted to get $Y^{\prime}$ and then the correlation between $X$ and $Y^{\prime}$ was measured. This was repeated 1000 times. The $p$ value is the fraction of random correlation strengths (between $X$ and $Y^{\prime}$ ), the absolute value of which are larger than the absolute value of the correlation between $X$ and $Y$.

Estimate of signal correlations between identical but noisy neurons. The maximal possible signal correlation between two identical but noisy neurons was estimated by what we call the "identical cell signal correlation." Instead of comparing the signals of two different neurons, the trials of a single neuron were divided into odd and even trials, the signal for each group of trials was determined by averaging across them, and signal correlations were calculated as described above. Again, only stimuli that elicited reliable responses were considered and at least 10 trials must have been recorded. A comparison between identical cell signal correlations and pairwise signal correlations is shown in Figure $6 E$.

Tests using bootstrapped signal and noise correlations. The confidence intervals of signal and noise correlations were determined by bootstrapping. For each pair of neurons, responses were sampled with replacement from as many trials as were recorded and then signal and noise correlations were determined as described above. This procedure was repeated 500 times. The bootstrapped distribution of a pair's signal correlations was used to determine whether they differ across bin sizes (KruskalWallis test with $p<0.05$ ) and whether they monotonically increase (or decrease) by checking that the signal correlation for bin size $i+1$ is not significantly smaller (or significantly larger) than that for bin size $i$ (using the comparison intervals resulting from Tukey's honestly significant difference criterion for multiple comparisons with a significance level of $5 \%)$. The confidence intervals of noise correlations determined from the bootstrapped distributions are shown in Figure $10 \mathrm{D}$ to demonstrate the robustness of their estimates. Furthermore, the bootstrapped distributions were used to get confidence intervals for the strength of correlation between noise correlations of different stimulus classes (Fig. 11), as well as between signal and noise correlations (Fig. 12B).

Control test for influence of time scale on signal correlations. When signal correlations were compared across bin sizes, differences could arise due to noisier estimates of firing rates on smaller bins compared with larger ones. To estimate the strength of this effect, data were simulated based on a fixed firing rate to which random noise was added in each trial. To measure the effect of different noise levels, data were simulated so that signal correlations on different bin sizes would be equal if no noise was added. Therefore, the mean firing rate that was measured on bins of 200 ms was used as the baseline rate. To simulate a neuron's response for a bin size of $10 \mathrm{~ms}$, the baseline rate was binned into $10 \mathrm{~ms}$ intervals and normally distributed noise was added to each bin. The magnitude of the noise depended on the variance of firing rate measured on the $10 \mathrm{~ms}$ bins. Because this variance increases linearly with firing rate, the two quantities were first related by a linear regression. Then the SD of the normally distributed noise could be determined by looking up the variance observed for the bin's firing rate. In this way, as many trials were simulated as were measured, and signal correlation was calculated between the simulated responses of two neurons as described above. Signal correlations for other bin sizes were simulated accordingly. These simulations show how much signal correlations are expected to change with bin size due to noise characteristics only.

\section{Results}

The analyses are based on recordings of 122 neurons in area 17 of 15 adult cats. Pairs of neurons $(n=46)$ and triplets $(n=3)$ were recorded with a single high-impedance pipette. An additional 21 single neurons were included in control statistics (for comparison of tuning differences between neighboring neurons with those between randomly chosen neurons, see following section). Figure 1 shows an example voltage trace containing spikes of three simultaneously recorded neurons (Fig. 1A), together with examples of overlapping spikes that could be distinguished in the spike-sorting procedure (Fig. $1 B$ ). Figure $1 C$ shows raster plots for the simultaneous responses of the three neurons to multiple trials of a drifting sinusoidal grating featuring the neurons' optimal orientation and direction, a monochrome natural movie scene, and binary dense noise stimuli consisting of high-contrast black-and-white bars presented at the optimal orientation. For 16 pairs of simultaneously recorded neurons, responses to all three stimulus classes could be recorded, whereas the remaining pairs were lost before the end of the stimulus protocol or were unresponsive to some of the stimuli.

An overview of the response measures we used to compare neighboring neurons is given in Figure 2. We started our investigation by comparing the tuning curves of neighboring neurons based on their responses to drifting sinusoidal gratings (Fig. 2, top). Next, we compared their "signals," which are their firing rates averaged across several repetitions of the same stimulus. Specifically, we measured the correlation between their signals in response to any of the three stimulus classes (Fig. 2, bottom). In contrast to tuning curves, signal correlations also compare responses at a finer time scale of tens to hundreds of milliseconds next to those measured on a coarse scale of several seconds (trial correlations), which allowed us to examine how different time scales affect the similarity of the neurons' signals. We then asked how well tuning differences in pairs of neighboring neurons relate to their signal correlations and what influence the stimulus class has on the strength of their signal correlations. As a measure of the degree of common input to the neurons, we analyzed the trial-to-trial fluctuations, termed "noise," in their responses to the same stimulus (Fig. 2, bottom). We quantified the strengths of these noise correlations, compared them between stimulus classes, and examined their relationship to the neurons' signal correlations. To validate our results, we controlled for the influence of firing rates on the magnitudes of and relations between the various correlations we investigated. Last, we compared all of our measures of response differences across cortical layers.

\section{Responses of neighboring neurons differ substantially \\ Tuning differences}

The first step in our comparison of neighboring neurons was to estimate the similarities of their RFs in response to drifting sine wave gratings. For each pair or triplet, we determined their preferences for orientation, direction, spatial and temporal frequency, their tuning widths for orientation and spatial frequency, their direction index (indicating how much more the neuron prefers motion into one direction over the opposite), and their preferred phase. Figure $3 A-C$ shows examples of tuning curves of the three simultaneously recorded neurons; their raw data are shown in Figure 1. The responses were fit with Gaussian functions or variations thereof. Figure $3 A$ shows that the orientation preferences of one neuron differed from the other two, but that all had the same direction preference. Direction indices ranged from 0.23 for the neuron indicated in orange, which had similarly strong responses to both directions, to 0.85 for the neuron indi- 


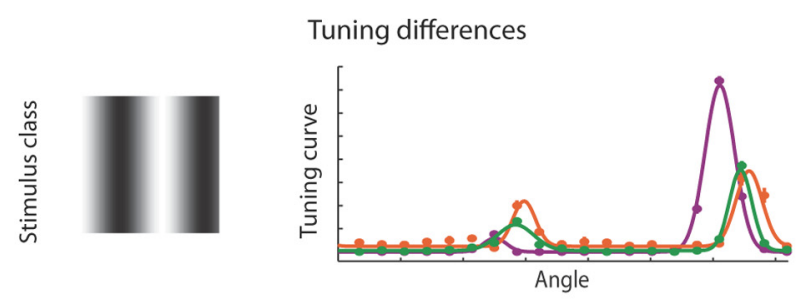

Signal and noise correlations
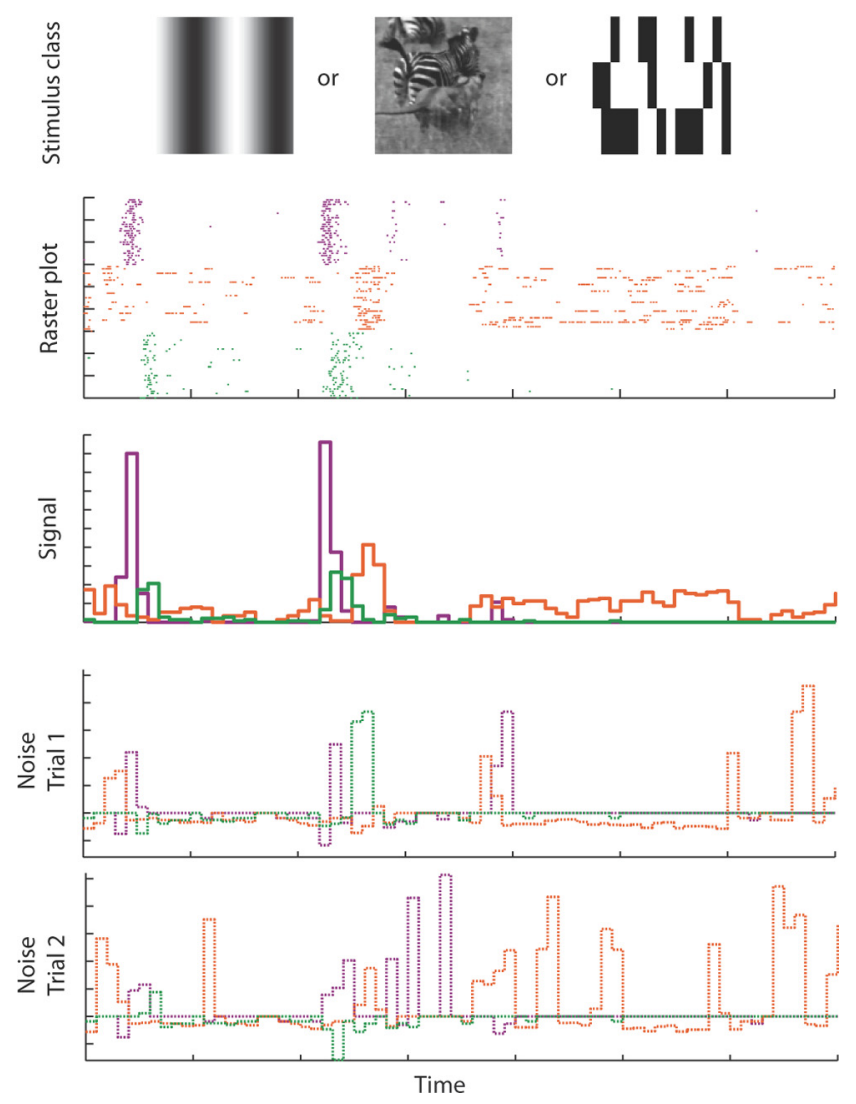

Figure 2. Overview of analyses. Top: Grating stimulus and tuning curves upon which the analysis of tuning differences are based. Bottom: Data used for signal and noise correlations: responses to all stimuli of one class were used; spike times (depicted in raster plots) were binned and averaged across trials to determine the signals of the neurons; deviations from this signal in each trial constitute the noise of each neuron.

cated in purple. All three neurons had similar spatial frequency tuning (Fig. 3B), but one cell differed markedly from the other two in its temporal frequency tuning (Fig. 3C). As can be seen in Figure $3 C$, we sampled temporal frequencies very finely, but, unfortunately, missed values high enough to get the full tuning range of the neuron with the purple curve. In such cases (see Materials and Methods), we did not consider the neuron's preference for temporal frequency. Therefore, our analyses on temporal frequency preferences are limited to lower values up to 2.5 cycles/s.

To determine the preferred phase of a neuron, its spikes were aligned to the start of each drift cycle of the grating stimulus. The preferred phase was then defined at the maximum of the first harmonic of the aligned spikes. The example neurons in Figure $3 D$ had very different phase preferences. Note that this definition of preferred phase incorporates spatial and temporal aspects of the neuron's phase preference. In contrast to stationary contrastreversal gratings, drifting gratings do not allow for a distinction between these two aspects. The greater part of phase differences we measured on the basis of our definition, however, can most likely be ascribed to differences in spatial aspects. DeAngelis et al. (1999) found that the preferred temporal phases in simple cells were confined to a narrow range, whereas preferred spatial phases were distributed uniformly over the range of possible values and contributed most to differences between spatiotemporal RFs of neighboring simple cells. Furthermore, our measure of phase difference does not take into account the distance between RFs. It cannot, for example, distinguish between two overlapping and structurally equal RFs that just differ in the polarity of their subfields (i.e., the off-region of one neuron overlaps with the onregion of the other neuron and vice versa) and two equal RFs shifted in space by the width of one subfield. However, phase differences, as we define them, do reflect absolute positional differences between the RFs' subfields that have the same polarity (on or off).

To compare the tuning across all neuron pairs and triplets, we measured the absolute differences between the tuning properties of neighboring neurons and compared these with the absolute differences that would be expected if RF properties were not clustered in cortex (Fig. 4). Expected differences were estimated by pairing randomly selected neurons from different recording sites (see Materials and Methods). Figure $4 A$ shows that the difference between the preferred orientations of two neighboring neurons (black bars) was often much smaller than that between two randomly selected neurons (red line). Similarly, preferred direction of movement (Fig. 4B) and tuning width for orientation (Fig. 4D) were significantly more similar in adjacent neurons than in two randomly chosen cells $(p<0.05$, permutation test, see Materials and Methods). To quantify the degree of clustering for each tuning property, we devised a measure previously introduced by DeAngelis et al. (1999) termed the "clustering index"' (see Materials and Methods). It is the ratio between the expected difference (the difference seen between randomly chosen neurons) and the observed difference between adjacent neurons (Fig. $4 J$ ). If the clustering index is larger than 1, neighboring neurons are more similar to each other than two randomly picked neurons. Again, preferred direction and preferred orientation were very strongly clustered within cortex. Although preferred temporal frequencies had a high clustering index, the neighboring neurons were not significantly more similar than expected. This is probably due to the small number of pairs. Furthermore, the differences in the chance distribution of preferred temporal frequencies were most probably underestimated, because we only sampled low values of temporal frequencies and missed neurons with higher preferences. Conversely, our estimate of differences between neighboring neurons is probably a fairly good description for the whole population of pairs, because data from DeAngelis et al. (1999) showed that differences in preferred temporal frequency between neighboring simple cells are fairly constant and independent of the absolute preferred values (Fig. $11 \mathrm{~F}$ in DeAngelis et al., 1999). We therefore expect that temporal frequency shows clustering in cortex when the complete range of preferences is considered. Other tuning properties, particularly direction index, tuning width for spatial frequency, and preferred phase (Fig. $4 E, F, H$, respectively), were randomly distributed among neighboring neurons. For these tuning properties, clustering indices were close to 1 and differences between neighboring neurons were not significantly smaller than differences between randomly selected neurons. These data suggest that some tuning properties do not cluster on a fine spatial scale within primary visual cortex. 
Having measured differences in single tuning parameters, we then investigated how differences across parameters are combined and whether some pairs of neighboring neurons have very similar tuning properties on the whole. To do this, we rescaled differences in each tuning property so that they were directly comparable to each other. For each tuning property, the differences between two cells were expressed in percentiles of the expected differences between two randomly selected neurons (Fig. 5A,B). Therefore, if the difference between two neighboring neurons falls into the $n^{\text {th }}$ percentile, then the cells are as similar as the $n \%$ of the most similar random pairs. The tuning differences of each recorded neuron pair are represented in Figure 5C. Differences for each tuning property are depicted in a different color, whereas a pair's mean difference across all properties is shown as a circle. The neuron pairs were ranked from the most similar to the most dissimilar pair. Only for four of 49 pairs were all measured tuning differences smaller than the $50^{\text {th }}$ percentile of the chance distribution. Most pairs had some tuning properties in common, but the great majority showed large differences in at least one tuning property.

Signal correlations in response to artificial and natural stimuli

The "signal" of a neuron is its purely stimulus-related response and is estimated by averaging the neuron's responses across trials assuming that deviations from that signal, commonly referred to as "noise," are independent across trials. The signal correlation of two neurons is thus a measure of the similarity between their stimulus-related responses. We calculated signal correlations here as Pearson's correlation (covariance divided by product of SDs) between each neuron's average firing rates in response to all presented stimuli of one class (gratings, movies, or visual noise; see Materials and Methods). We measured firing rates on various bin sizes to assess the influence of different time scales on the strength of signal correlations. Because the calculation of signal correlation depends on a good estimate of the average firing rates of each neuron, we only considered responses to stimuli if both neurons fired in a fairly reliable manner across trials (see Materials and Methods). First, we considered signal correlations in response to gratings. In Figure 6A, firing rates of two adjacent cells were assessed over the complete duration of a grating stimulus lasting for $5 \mathrm{~s}$. Each dot represents the mean responses of both neurons and is colored according to which stimulus parameter (orientation, spatial frequency, or temporal frequency) was varied. To avoid spurious signal correlations, firing rates in response to variations of a single stimulus parameter were $z$-transformed (mean subtracted and divided by SD) before the signal correlation on all transformed responses was determined (see Materials and Methods). We will from here on refer to this measure as "trial correlation," because firing rates were estimated from complete trials as supposed to smaller time bins,
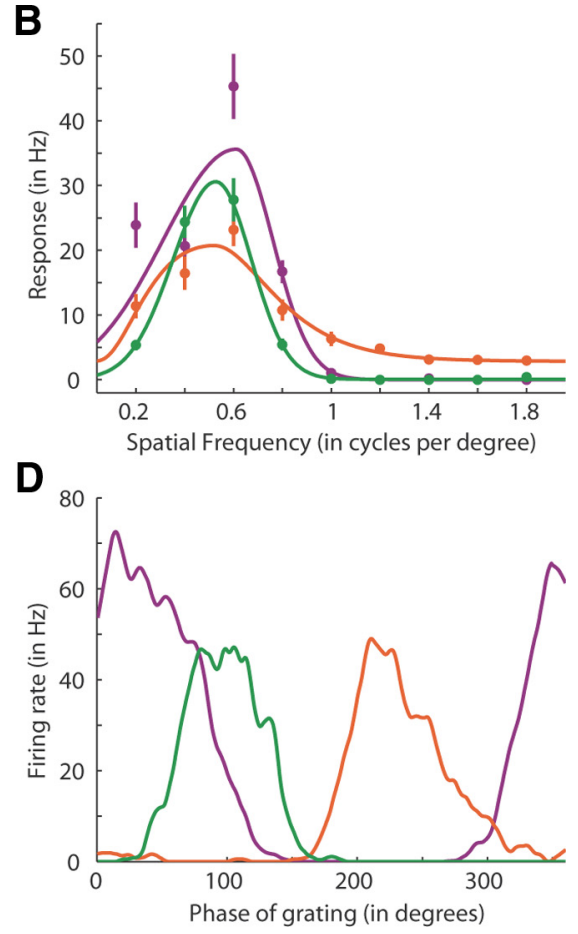

Figure 3. Tuning curves and phase modulation of the same three simultaneously recorded neurons in Figure 1 (same colors for neuron identity). $\boldsymbol{A}-\boldsymbol{C}$, All tuning curves of the neurons were based on their median responses across all repetitions of each grating (dots). In this case, the RFs of all three neurons were classified as simple, so the response refers to the amplitude of the first fic of the spike pattern in response to one cycle of the grating (F1 responses). Error bars show the mean absolute deviation fesponse divided by the square root of the number of trials (see Materials and Methods). $A$, Responses of each better fit determined which of the two options was chosen. $C$ Responses to different temporal frequencies of the gratings were fit with a Gaussian (purple and green curves) or a logarithmic Gaussian (orange curve). $\boldsymbol{D}$, Instantaneous firing rate of each neuron during the course of one drift cycle of the grating.

which we will investigate in the following paragraphs. The example shown in Figure $6 A$ shows a pair with a fairly high positive trial correlation of $0.53(p<0.001)$. The population results (Fig. $6 B$ ) show that $48 \%$ of the pairs of neighbors (12 of 25 pairs) had positive trial correlations larger than 0.5 . The median trial correlation across all pairs was 0.48 , indicating a high heterogeneity in response properties between neighboring neurons.

The differences between neighboring neurons grew even larger when their responses were compared on finer temporal scales. Signal correlations determined from firing rates on small bins ranging from 10 to $200 \mathrm{~ms}$ were used to quantify such differences. An example for two neurons is shown in Figure 6C, which plots the average responses in bins of $50 \mathrm{~ms}$ to repetitions of a $10 \mathrm{~s}$ movie sequence. To assess the signal correlation of the cells, their binned firing rates in response to all movies (in this case, two movies) were correlated with each other (Fig. 6D). In this pair, the signal correlation for movies had a very high value of $0.81(p<0.001)$. Across all neuron pairs, however, the median signal correlation was always close to zero and the absolute strength of signal correlation for $50 \%$ of the pairs (interquartile range) stayed well below 0.5 , no matter which stimulus class was presented (Fig. 6E). Signal correlations in the same range were seen for neighboring neurons in cat area 17 in response to movies (Yen et al., 2007), but were higher in V1 of anesthetized or awake monkeys in response to visual noise stimuli (Gawne et al., 1996; Reich et al., 2001). 

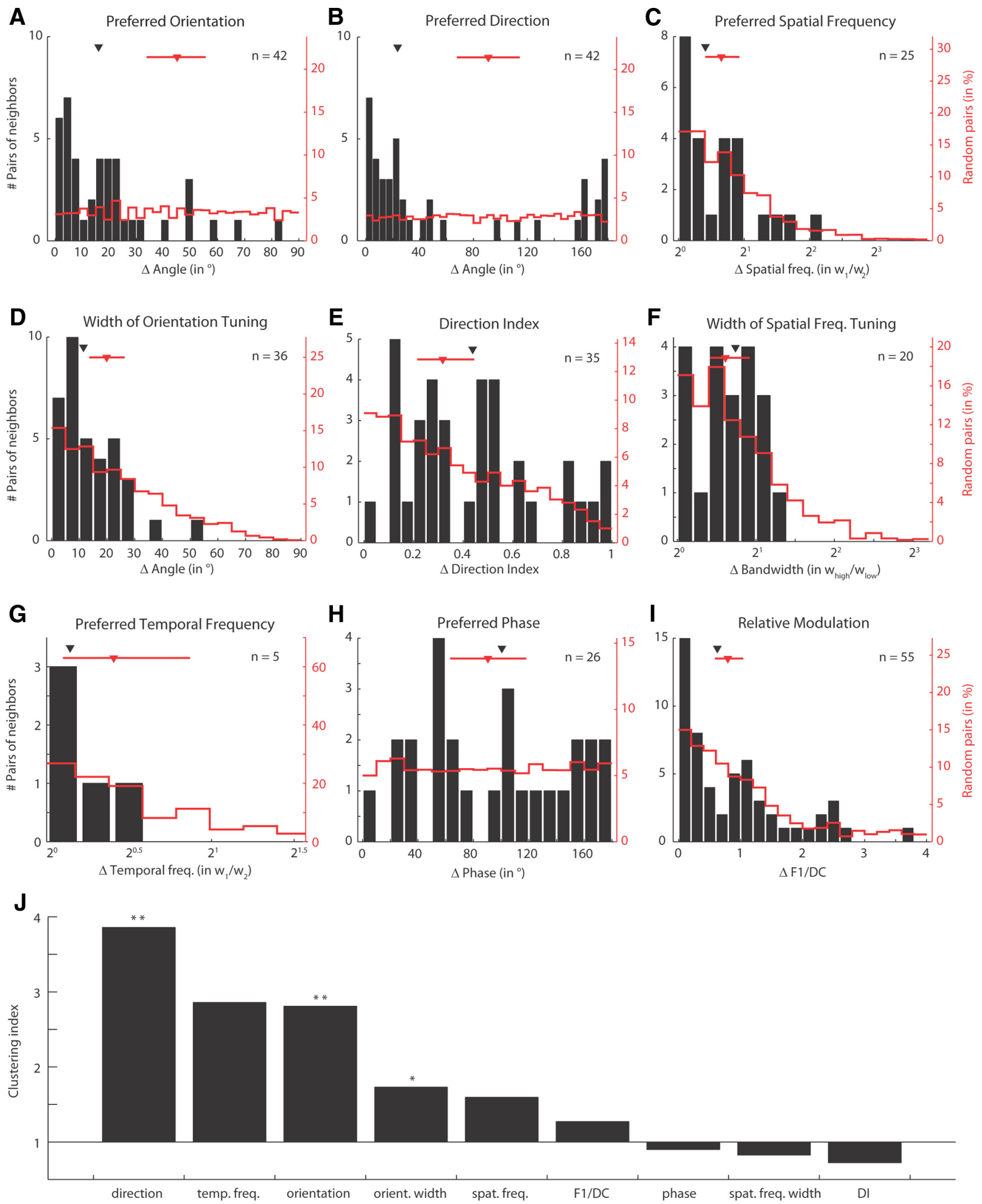

Figure 4. Comparison between tuning differences of neighboring neurons and those of randomly picked neurons. $A$, Distribution of differences between the preferred orientations of two neighboring neurons (black bars); left $y$-axis indicates the number of observed pairs. Black triangle at the top indicates the median difference. Red outline shows distribution of differences in pairs of two randomly selected neurons that were not simultaneously recorded (red $y$-axis to the right depicts percentage of these pairs; scales of red and black $y$-axes are equivalent). This distribution resulted from pooling all 1000 random distributions (see Materials and Methods). Red triangle indicates the median of all random medians, red horizontal line the confidence interval (5-95\%). $\boldsymbol{B}-\boldsymbol{I}$, Same as in $\boldsymbol{A}$ but for different tuning properties. In each panel, the number of pairs that were considered is given by $\boldsymbol{n}$. Not all tuning curves could be measured for all pairs and not all tuning properties could be determined from the neurons' responses, so $n$ differs across the stimulus parameters.J, The clustering index (see Results) for each stimulus parameter is depicted. Two stars indicate a highly significant difference ( $\boldsymbol{p}<0.01$, permutation test) between the actual and the chance distribution of differences between neurons, one star indicates a significant difference $(p<0.05)$. 

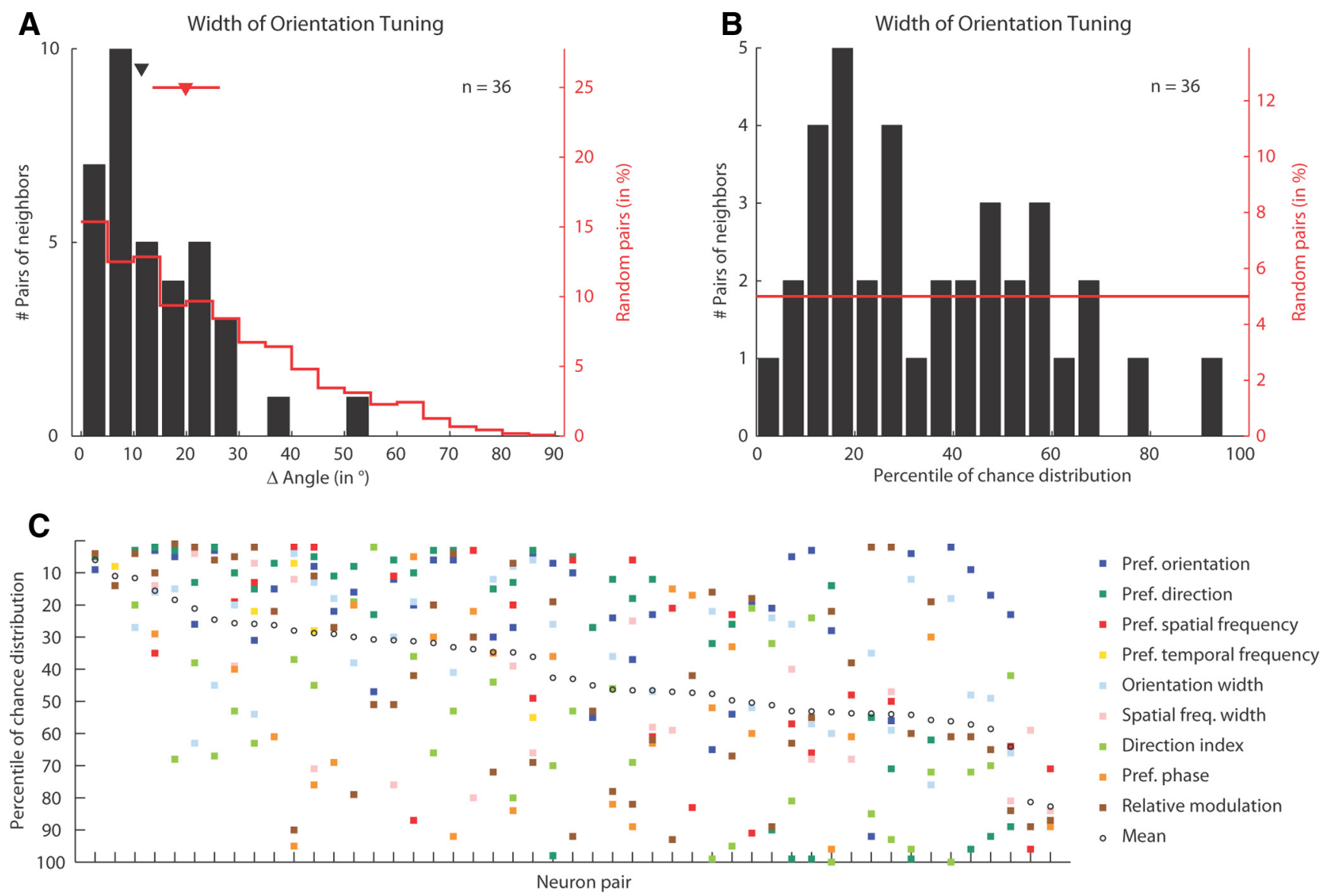

Figure 5. Tuning differences between neighboring neurons across all stimulus parameters. $A$, Same plot as in Figure $4 D$. Differences between the neurons' width of orientation tuning is given in degrees. Black bars depict differences between neighboring neurons; the red line differences between randomly chosen neurons. $B$, Same data as in $\boldsymbol{A}$, but now differences between the neurons' tuning width (black bars) are given in percentiles of the chance distribution (i.e., in terms of the differences between randomly picked neurons; see Results). The distribution of differences between randomly chosen neurons (chance distribution; red line) is therefore flat. $\boldsymbol{C}$, Tuning differences for all stimulus parameters plotted for each pair of neighboring neurons expressed in percentiles of the chance distribution as in $\boldsymbol{B}$. Neuron pairs were ranked with the most similar pair (i.e., the one with the lowest mean tuning difference across all measured parameters) at the left. Color of each square refers to the stimulus parameter. Mean differences are depicted as black circles.

The strength of signal correlation for two neurons depends on how well the average firing rates of the neurons can be estimated and ultimately on how noisy their responses are. We thus compared the signal correlations measured for pairs of neighboring neurons with signal correlations that are expected in identical but noisy neurons. These reflect the maximal strength of signal correlation that can be reached by two neurons given the level of noise. We estimated this by dividing the trials of a single neuron into two groups as if they originated from two different neurons (see Materials and Methods). Given that the neuron was recorded for a sufficient number of trials, the correlation between the average firing rates of each of the two trial groups is an estimate of the signal correlation between identical neurons. The median of these "identical cell signal correlations" across all single cells is depicted as diamonds in Figure $6 E$ for each bin size and stimulus class. The noise in single cell responses dramatically lowered the expected signal correlation for identical neurons, specifically for the slowly varying gratings and the smallest bin size of $10 \mathrm{~ms}$ (median of 0.177 ). Nevertheless, the median "identical cell signal correlation" was always larger than $80 \%$ of the pairwise signal correlations; in the case of movies and visual noise, it was exceeded by at most two out of 18 or one out of 15 pairwise signal correlations, respectively. Signal correlations in neigh- boring neurons were much lower than can be explained by the noise in the neurons' individual responses.

\section{Influence of time scale on signal correlations}

Although the median of signal correlations stayed close to zero for bin sizes from 10 to $200 \mathrm{~ms}$, their range increased. When we examined signal correlations for each pair separately (data shown in Fig. $7 A-C$ ), we found that their absolute strengths increased significantly with increasing bin sizes from 10 to $200 \mathrm{~ms}$ in 27 of 65 recordings (taken from 37 pairs) in response to gratings, movies, or visual noise (considering only recordings, for which signal correlations on $200 \mathrm{~ms}$ and at least one smaller bin size existed). Signal correlations for these recordings were significantly different between bin sizes $(p<0.05$, Kruskal-Wallis test on bootstrapped signal correlations; see Materials and Methods), the signal correlation on the largest bin (200 ms) was significantly different from zero $(p<0.05$, permutation test), and there was a monotonic increase or decrease of signal correlations with increasing bin sizes (see Materials and Methods). Figure 7D, E (red dots) shows two pairs with a significant negative and positive trend, respectively, whereas the pair in Figure $7 F$ (red dots) shows no monotonic trend, as was the case in seven of 65 recordings. In the remaining recordings ( 31 of 65), signal correlations on 200 ms bins were not significant. 
A
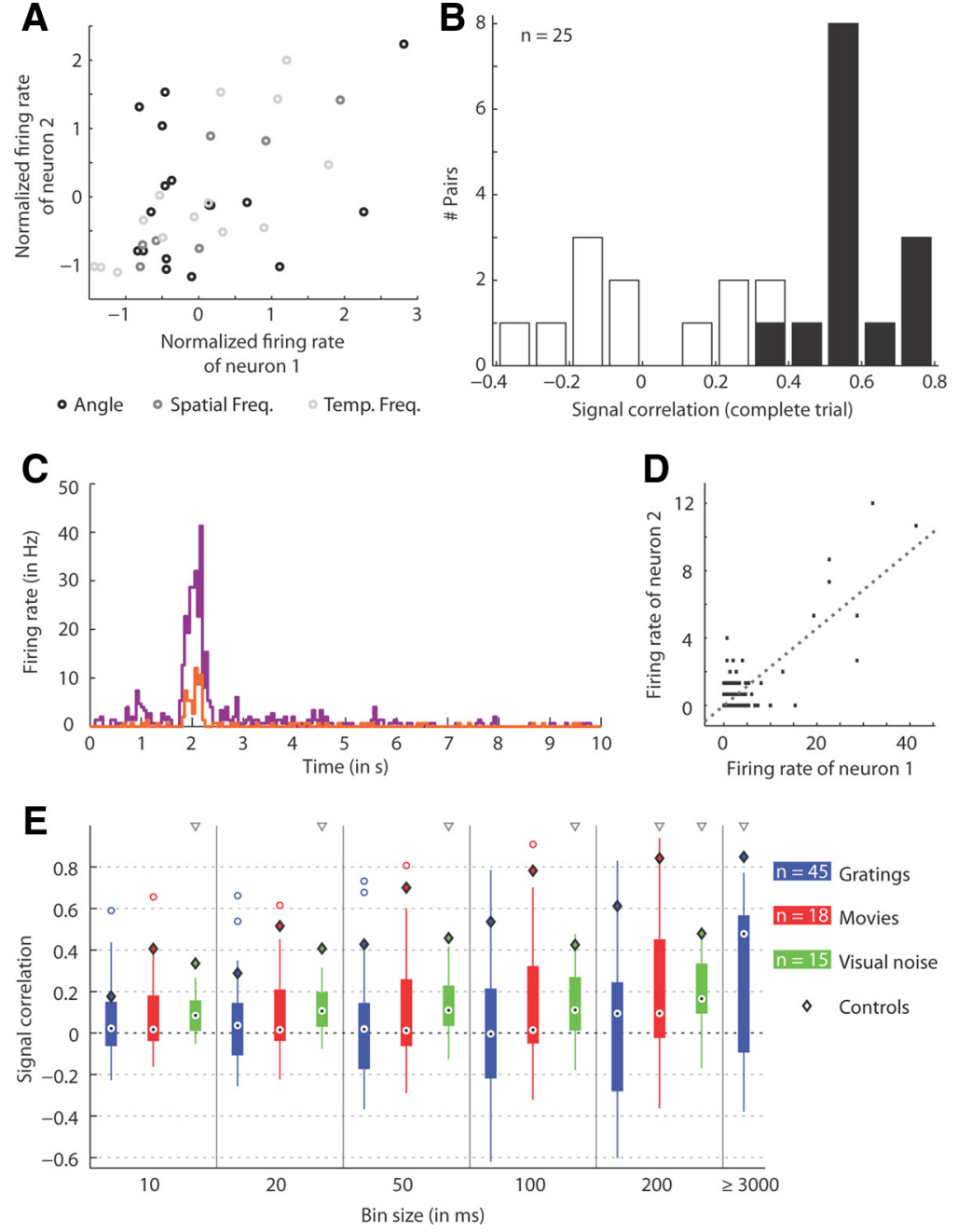

Figure 6. Signal correlations in response to artificial and natural stimuli. $\boldsymbol{A}$, Firing rates in response to gratings measured on complete trials and averaged across all presentations of each grating stimulus are plotted for two simultaneously recorded neurons (cat0210 P3C4). The firing rates in response to gratings varying in one stimulus feature, namely in orientation and direction (black), spatial frequency (dark gray), or temporal frequency (light gray), were $z$-transformed separately (see main text). The signal correlation in this example was $0.53(p<0.001) \boldsymbol{B}$, Histogram of signal correlations for gratings (complete trials) for all $n$ pairs of neighboring neurons. Black bars indicate significant correlations $(p<0.05)$, white bars nonsignificant correlations. C, Firing rates of the same two neurons as in $\boldsymbol{A}$ (purple and orange trace, respectively) in response to a 10 -s-long movie sequence. Spikes were binned into $50 \mathrm{~ms}$ intervals and firing rates averaged across 30 presentations of the same movie. $\boldsymbol{D}$, Firing rates of both neurons plotted against each other for each time bin in Cas well as for data from a second movie presented to the same pair of neurons. The signal correlation in this example was $0.81(p<0.001)$. Dashed line represents the linear regression line. $\boldsymbol{E}$, Signal correlations for all three stimulus classes, gratings, movies, and visual noise, determined using various bin sizes. For comparison, the distribution of signal correlations measured on mean spike counts of complete trials ( 3 or 5 in duration) is also included (rightmost box). The boxes extend from the $25^{\text {th }}$ to the $75^{\text {th }}$ percentiles; the median is indicated by the black dot. Whiskers show the whole range of signal correlations, whereas outliers (further than 1.5 times the box length away from the box edge) are marked by circles. Distributions in which medians were significantly different from zero are labeled with a triangle above the box $(p<0.05$, signed-rank test). Diamonds represent median signal correlations expected from identical but noisy neurons (see Materials and Methods and Results).

The significant trends seen in some of the recordings might reflect a dependence of signal similarity between neighboring neurons on the temporal stimulus statistics. A second possibility is that firing rates estimated on smaller bins are more susceptible to noise in the neuron's responses than those estimated on larger bin sizes. The increase of absolute strength of signal correlations might therefore be explained by varying noise statistics across time scales rather than by the temporal stimulus statistics. To test for this, we generated neural responses from an assumed "true" signal emitted in response to the stimulus and additional noise with a magnitude depending on the considered bin size. The assumed true signal was taken from the neuron's mean firing rate measured on bins of $200 \mathrm{~ms}$, because these estimates were the least variable, and was then parceled into smaller bins if necessary. The noise that was added was based on the measured variance of firing rate for each bin size (see Materials and Methods). If no noise had been added to the simulated neural responses, signal correlations would have been the same across all bin sizes. Therefore, any differences in correlations of the simulated signals that are observed between different bin sizes can be attributed to the different noise levels and the different numbers of bins used to generate the signals. Examples of signal correlations based on simulated data compared with the measured signal correlations are given in Figure $7 D-F$ (gray boxes). The examples show that simulated signal correlations decreased in absolute strength the smaller the bin size was (i.e., the more noise was added to the original signal). To assess the strength of the trend in simulated and measured signal correlations, we calculated the normalized difference between the signal correlation on 200 ms bins, $S C_{200}$, and on a smaller bin size, $S C_{\mathrm{b}}$ as follows:

$$
\left(S C_{200}-S C_{b}\right) /\left(S C_{200}+S C_{b}\right)
$$

We refer to this measure as the "trend index." The measured trends of signal correlations in the pairs shown in Figure 7D, $E$ were much stronger than those from the simulated responses for all bin sizes (compare red dots with gray boxes in Fig. $7 G$, $H)$. This shows that the trend of increasing strengths of signal correlations alone cannot be explained by the varying noise statistics across bin sizes. The same was true for approximately $30 \%$ of all recordings in which a positive or negative trend was observed ( 8 of 27; Gratings: three with positive and two with negative trends, Movies: three with positive trends; $p<$ 0.05, two-way ANOVA with measured and simulated trend indices as one factor and bin size as second factor). In contrast, signal correlations at larger time scales of several seconds that we measured in response to gratings were not significantly correlated with those at smaller time scales $(p>0.16$ for all bin sizes, permutation test).These results show that the time scale at which signals are compared between neurons does matter and should be chosen with care. The reasons for a change in signal correlations with a change in time scale probably lie in the interplay between differences in the neurons' RFs and the spatiotemporal stimulus statistics. If a stimulus causes two neurons to respond with an elevated firing rate during approximately the same extended time periods but without high precision, they will have a low signal correlation 
on small time scales, which then increases on larger bin sizes. Examples of this are the neurons indicated with purple and green spikes in Figure $1 C$ responding to the movie. In the opposite case, namely when the neurons fire during nonoverlapping or almost nonoverlapping extended time periods (as the neurons indicated with purple and orange spikes in Fig. $1 C$ responding to gratings), their signal correlations will have a negative trend with increasing time scale.

\section{Relation between tuning differences and signal correlations}

Our data indicate that neighboring neurons have low signal correlations for a variety of stimuli. Furthermore, only a few tuning properties, preferred orientation, direction, and width of orientation tuning, seem to be similar between neighboring neurons. But how much can differences between neighboring neurons in such classic tuning parameters tell us about their signal correlations measured in response to various stimulus classes? Our data give only an approximate answer to this question, because the analyses required not just the assessment of tuning differences, but also the responses to movies or visual noise. These analyses could only be made for a relatively small number of neuron pairs. The correlation strengths between differences in tuning characteristics and the pairs' signal correlations are plotted in Figure $8 \mathrm{~A}$. All tuning differences except RF offsets (see below) were measured in percentiles of the chance distribution (i.e., the distribution of differences between any two neurons in primary visual cortex; see above and Fig. $5 A, B)$. A number of tuning characteristics, preferred orientation, preferred spatial frequency, and direction index, showed a relatively high and significant relationship to trial correlations in response to gratings explaining $27-50 \%$ of the variance (Fig. 8A, filled blue squares). This result is not unexpected because these tuning parameters have the strongest influence on the shape of the neurons' tuning curves and, therefore, largely determine their trial correlations. Note, however, that signal correlations are calculated from mean firing rates whereas tuning curves are based on the neurons' F1 or DC responses according to their RF type (see Materials and Methods). The scatter plots of these data (tuning differences vs trial correlations of each pair of neighboring neurons) are shown in Figure $8 B-D$. Signal correlations measured on shorter time scales had almost no significant correlations with tuning differences. The only exception to this were signal correlations in response to movies measured on bin sizes of 100 and $200 \mathrm{~ms}$, which were significantly correlated with differences in relative modulation (Fig. $8 A$, filled red circles, column F1/DC). This means that if the neurons were phase modulated to a similar degree in response to drifting gratings, they would also respond more similarly to movies. The scatter plot for all pairs is shown in Figure 8E. Although differences in preferred phase seem to be highly correlated with signal correlations in response to visual noise, the low " $n$ " (in this case, only nine to 10 pairs) did not lead to significant results. Differences in tuning width for orientation or spatial frequency (not shown in Fig. 8A) had no significant relation to signal correlations.

A further difference between RFs that could substantially influence the strength of signal correlations is the offset between RFs, which we here measured as the distance between both neurons' RF centers. The RF centers were estimated from the responses to visual noise, which were used to reconstruct spatiotemporal RFs by determining either the spike-triggered average or the eigenvectors of the spike-triggered covariance (see Materials and Methods). The reconstructed spatiotemporal RFs were then fit to an RF model to determine the center of the RF. In this way, we measured the position of the RF centers of neurons in 13 pairs, which showed RF center offsets of between 0.02 and 1.4 visual degrees (median offset was 0.32 visual degrees). The last column in Figure $8 A$ shows that only signal correlations in re- 

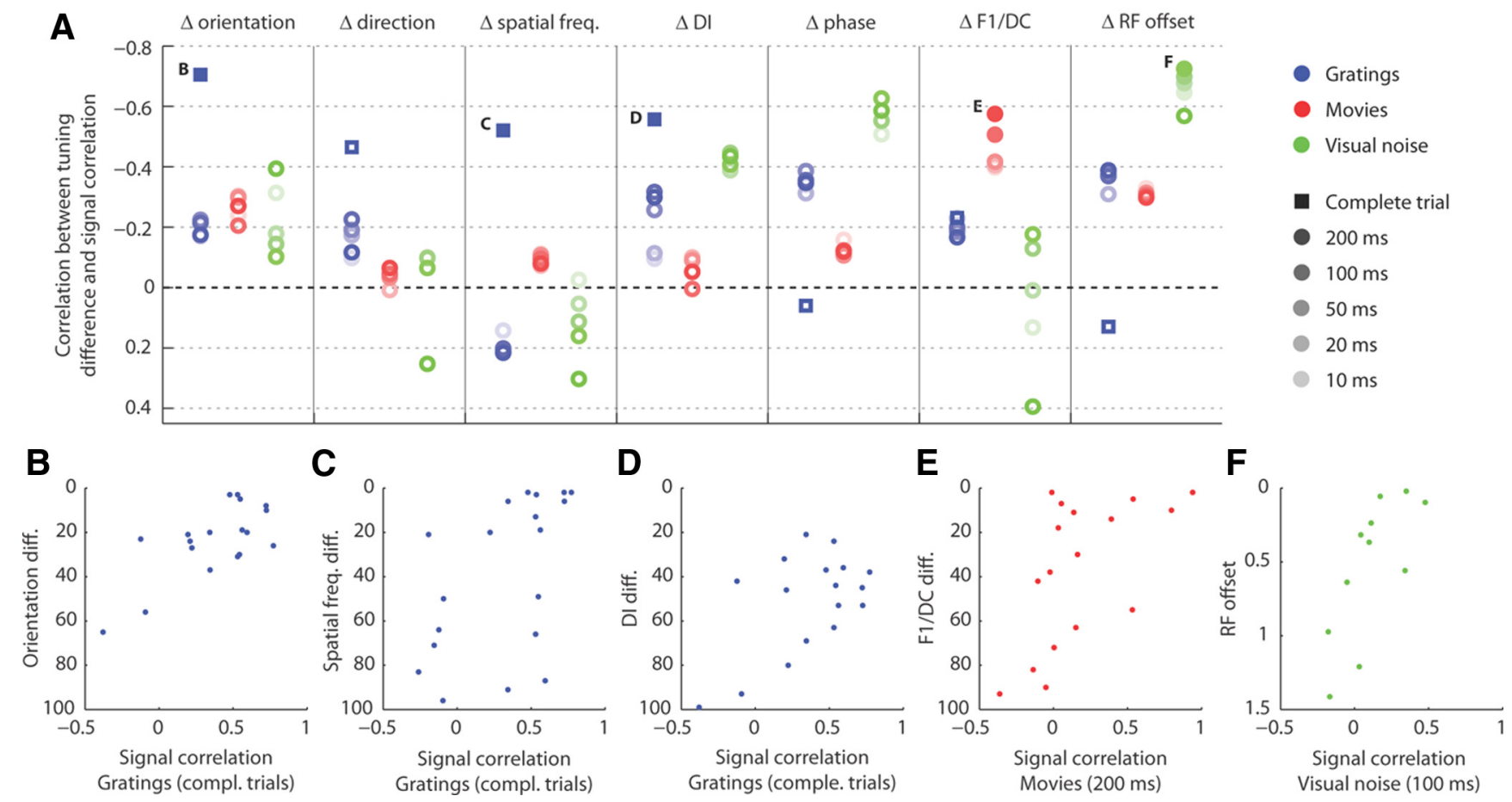

Figure 8. Correlation strength between tuning differences and signal correlations. $A$, Plot shows the correlation strengths between the pairs' signal correlations and their tuning differences in single preferred stimulus parameters, as well as in direction index, in relative modulation, and their RF offset (shown in different columns). The $y$-axis signifying the correlation strengths is reversed, because a close relationship between tuning differences and signal correlations would result in negative correlation coefficients. Tuning differences in each parameter were related to signal correlations for different stimulus classes (blue, red, and green) and for various bin sizes (the lighter the color, the smaller the bin size). Note that signal correlations on complete trials were only measured for grating stimuli (blue squares). Filled symbols mark significant correlations $(p<0.05)$. The letters next to some points mark significant correlations between tuning differences and signal correlations and refer to the plots, $\boldsymbol{B}-\boldsymbol{F}$, which show the underlying data. $\boldsymbol{B}$, Signal correlations measured on complete grating trials are plotted against the neurons' differences in preferred orientation. The correlation strength between both measures is depicted in $\boldsymbol{A}$ (square marked with " $\boldsymbol{B}$ "). $\boldsymbol{C}-\boldsymbol{F}$, Same as in $\boldsymbol{B}$ but for other tuning parameters and signal correlations on other bin sizes and for different stimulus classes (see label of axes).

sponse to visual noise were significantly correlated with these offsets (Fig. $8 F$ ). In summary, most tuning differences we considered here were more closely related to trial correlations, which were measured in response to drifting gratings on a time scale of several seconds. At smaller time scales, signal correlations had very weak relationships to most of the tuning differences and the strength of this relationship varied across stimulus classes.

\section{Signal correlations are similar for different stimulus classes}

In the previous section, we showed that differences between neighboring neurons in most tuning parameters poorly predict signal correlations on short time scales for any of the three stimulus classes. We now turn to the question of whether the neurons' signal correlations are similar across the different stimulus classes we used, gratings, movies, and visual noise. Any differences that occur will necessarily be caused by the different stimulus statistics. Full-field gratings, for example, have only one spatial dimensional (luminance along the second dimension does not change), whereas natural movies are two-dimensional in space. If the responses of neurons were sufficiently described by a linear RF the shape of a Gabor function, which is itself one-dimensional in space, signal correlations in response to gratings and movies should be approximately similar. However, there are many parameters that are not captured by the one-dimensional linear RF, such as surround effects or the degree of contrast adaptation, which may differentially modulate the responses of both neurons and lead to differences in signal correlations across stimuli. Furthermore, some differences between the neurons' RFs may be re- vealed by one but not the other stimulus class (e.g., those due to different temporal and spatial resolutions). Therefore, neurons are not expected to have similar signal correlations in response to different stimulus classes. Note, however, that the converse argument is not valid: similar signal correlations across different stimulus classes are not proof of linear RFs.

Our results show that signal correlations for different stimulus classes were strongly related to each other (Fig. 9). Regardless of the bin size used, correlation coefficients were between 0.58 and 0.81 when signal correlations for movies were compared with those for gratings or visual noise stimuli (Fig. 9A,C). They were even higher, between 0.8 and 0.91 , when signal correlations for gratings and noise stimuli were compared with each other (Fig. $9 B$ ). Below we will show that this agreement cannot be explained by firing rates of the pairs alone. In addition, we found no systematic differences between the strengths of signal correlation in response to different stimulus classes ( $p>0.06$, pairwise signedrank test). From these results, we conclude that the similarity between the stimulus-dependent responses of two neighboring neurons is largely independent of the stimulus class. In particular, even simple artificial gratings elicit average responses that are as similar or different between neighboring neurons as their responses to movies or visual noise stimuli.

\section{Noise correlations are small but similar across different stimulus classes}

In contrast to a neuron's signal, which reflects its stimulusdependent response, its trial-to-trial fluctuations in response to the same stimulus are commonly referred to as noise. Noise cor- 

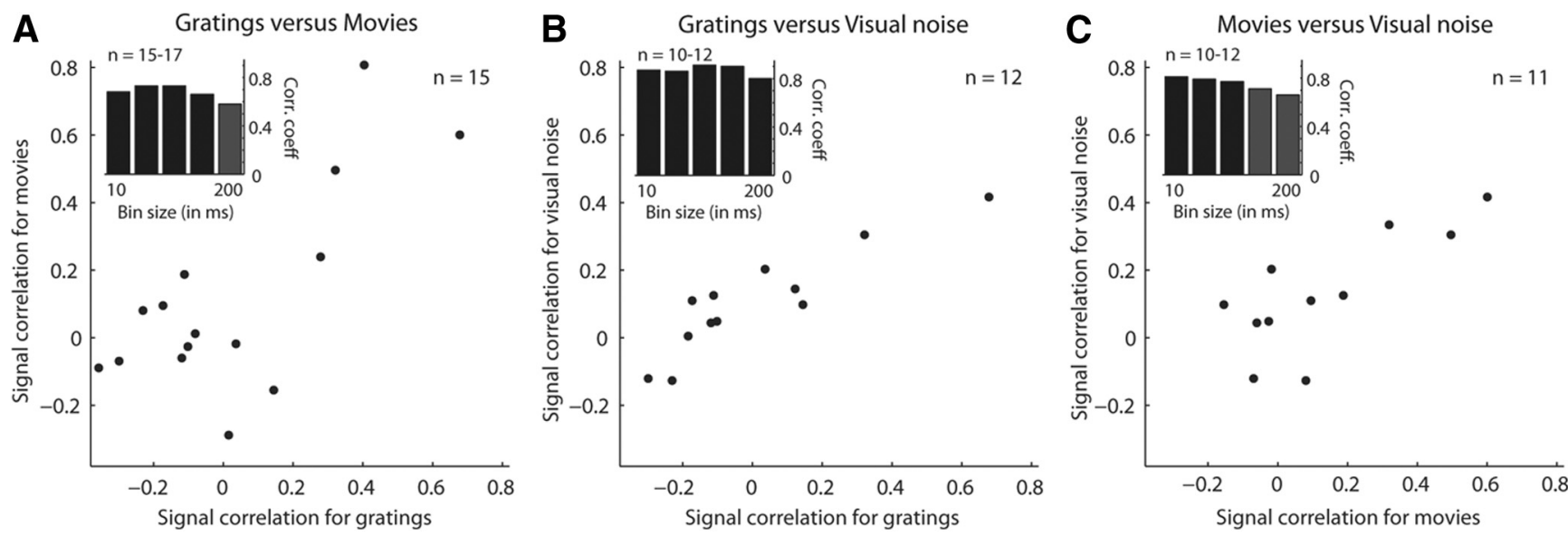

Figure 9. Correlation strengths between signal correlations of different stimulus classes. $\boldsymbol{A}$, Signal correlations measured on responses to gratings and to movies are plotted against each other for each pair of neighboring neurons. The responses were binned in intervals of $50 \mathrm{~ms}$. Inset shows the correlation coefficients between signal correlations using bin sizes of 10, 20,50, 100, and 200 $\mathrm{ms}$, respectively. $\boldsymbol{B}, \boldsymbol{C}$, Same as in $\boldsymbol{A}$ but for signal correlations measured on responses to different stimulus classes. Black bars in the insets signify highly significant correlations ( $p<0.01)$, gray bars significant correlations $(p<0.05)$; $n$ is the number of pairs.

relations measure the covariation of trial-to-trial fluctuations of two neurons independently of the stimulus; they are thought to arise primarily from inputs shared between the neurons (Bair et al., 2001; Kohn and Smith, 2005; Smith and Kohn, 2008). Although noise correlations cannot be used to infer how many inputs the neurons share in absolute terms (see Discussion), a comparison between stimulus classes can still tell us whether the amount of shared input changes. We computed the noise correlation of a neuron pair based on each neuron's deviation from its mean response to the stimulus divided by the SD. As for the case of signal correlation, only reliable responses of the neurons were taken into account (see Materials and Methods). Pearson's correlation was then calculated on the normalized spike count deviations pooled across all stimuli of the same class (see Materials and Methods). Because time scales of noise correlations in cortex were estimated to range from tens of milliseconds (Bair et al., 2001) to hundreds of milliseconds (Reich et al., 2001; Kohn and Smith, 2005; Mitchell et al., 2009), we considered spike counts on bins of 10-200 $\mathrm{ms}$ and, in response to gratings, of several seconds.

Figure 10 shows the noise correlations between adjacent neurons for the three stimulus classes. Figure $10 \mathrm{~A}$ depicts an example of deviations from the mean responses of two neighboring neurons for two repetitions of a movie scene and Figure $10 B$ shows the pair's noise correlation for the two different movie scenes that were presented during the recording. We found that across the whole population of pairs of neighboring neurons, median noise correlations were generally small, always staying below a value of 0.08 for bin sizes up to $200 \mathrm{~ms}$, regardless of stimulus class (Fig. $10 C)$. Because noise correlations depend on a good estimate of the neurons' mean firing rates and because the number of trials for some stimuli was relatively small in our dataset, we measured how robust our estimates of noise correlations were by performing a bootstrap analysis. Bootstrapped noise correlations were calculated from responses of randomly sampled trials (see Materials and Methods). Their relation to measured noise correlations is shown in Figure $10 D$ for bin sizes of $10 \mathrm{~ms}$. The medians of the bootstrapped noise correlations were very similar to the measured values for all stimulus classes and the 95\% confidence intervals in most cases were small, demonstrating the robustness of the measurements (larger confidence intervals in case of gratings are most likely due to smaller numbers of samples used to calcu- late noise correlations; Fig. 10D, legend). The range of noise correlations in our dataset is consistent with several previous studies (Bair et al., 2001; Reich et al., 2001; Kohn and Smith, 2005; Mitchell et al., 2009), as is the small trend of increasing noise correlations with increasing bin sizes from 10 to $200 \mathrm{~ms}$ visible in Figure $10 C$. When measured on long time scales of several seconds in response to gratings, the median noise correlations reached a value of 0.16 . Figure $10 E-G$ shows that noise correlations of several pairs increased with bin size, but decreased for only very few of them. Noise correlations measured on any two different bin sizes (between 10 and $200 \mathrm{~ms}$ ) were highly correlated with each other (pooled across stimulus classes, $\rho>0.7, p<0.001$, permutation test); those measured on complete grating trials were less well related to noise correlations at smaller time scales ( $\rho=0.36$, $p=0.06$ for 10 ms bins; $\rho=0.65, p<0.001$ for $200 \mathrm{~ms}$ bins $)$. Bair et al. (2001) showed that noise correlations are equivalent to the integral of the neurons' cross-correlogram (CCG) normalized by their auto-correlograms when limits of integration match the bin size of the noise correlations. Noise correlations in their recordings had the smallest variance across stimuli when the integration limits just enclosed the peak of the CCG but not its variable flanks. The CCGs of our pairs that showed signs of significant correlations had, on average, very narrow peaks extending over delays of 10-20 ms (data not shown). Accordingly, the noise correlations we measured on bins of 10 and 20 ms yield the best (i.e., the most robust) estimates.

Noise correlations in response to different stimulus classes were similar to each other. A comparison between noise correlations for gratings and movies showed that they were highly correlated with each other (Fig. 11). Noise correlations in response to visual noise were somewhat less well related to noise correlations in response to the other stimulus classes (specifically for bin sizes of 10 and $20 \mathrm{~ms}$ ), and this relation did not always reach a $5 \%$ significance level due to the small number of data points. Underlying these differences might the faster temporal dynamics of visual noise stimuli (20 ms frame rate) and their high contrast changes from frame to frame. The more rapidly changing firing rates (Fig. 1C) might influence both the dynamics of the neural noise response and the accuracy of its estimation. However, none of the stimulus classes led to consistently larger noise correlations ( $p>0.08$ for any bin size, pairwise signed-rank test). In the next section, we investigate the degree to which stimulus- 

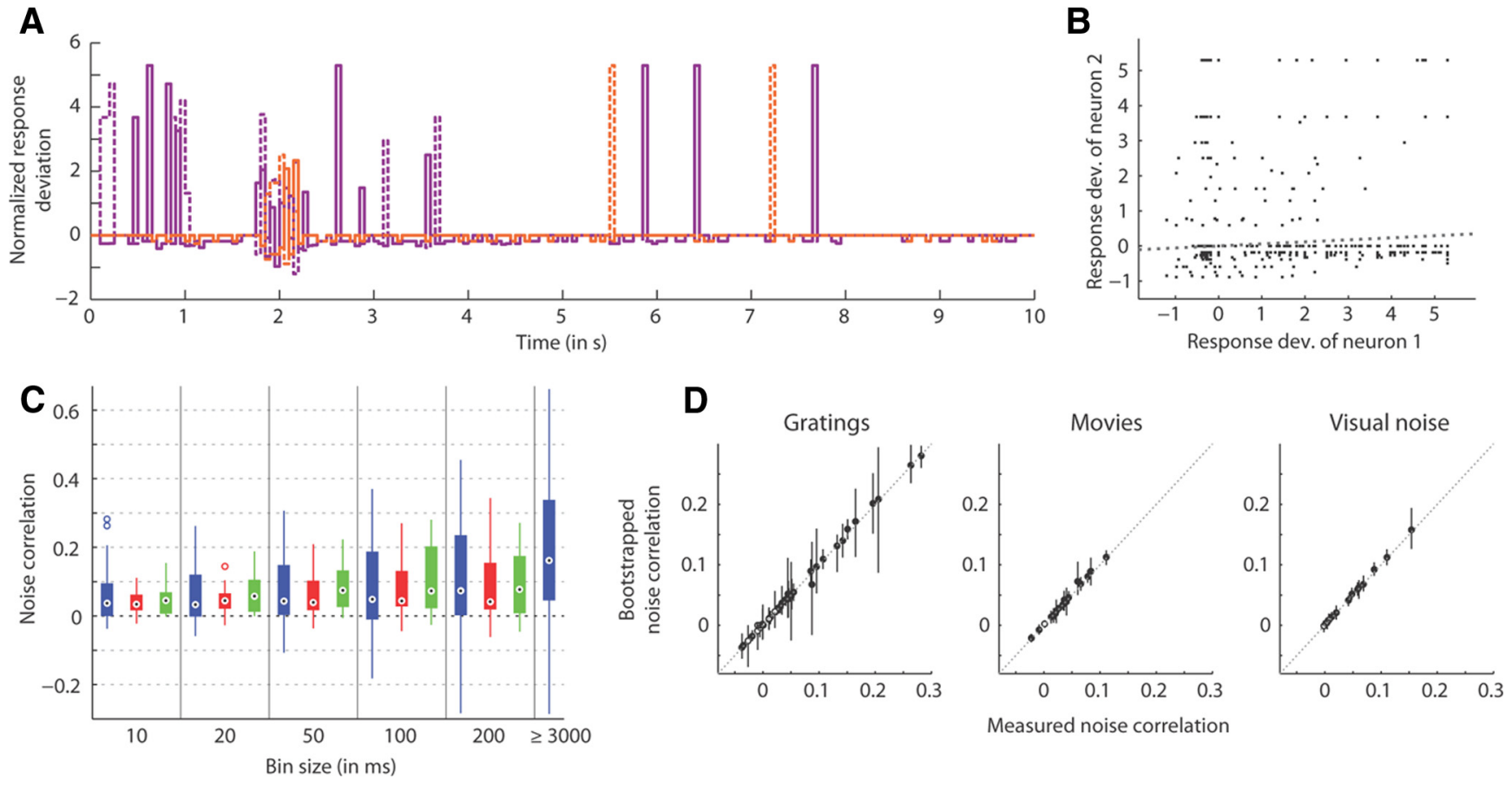

$\mathrm{n}=47$ Gratings $\mathrm{n}=18$ Movies $\mathrm{n}=15$ Visual noise
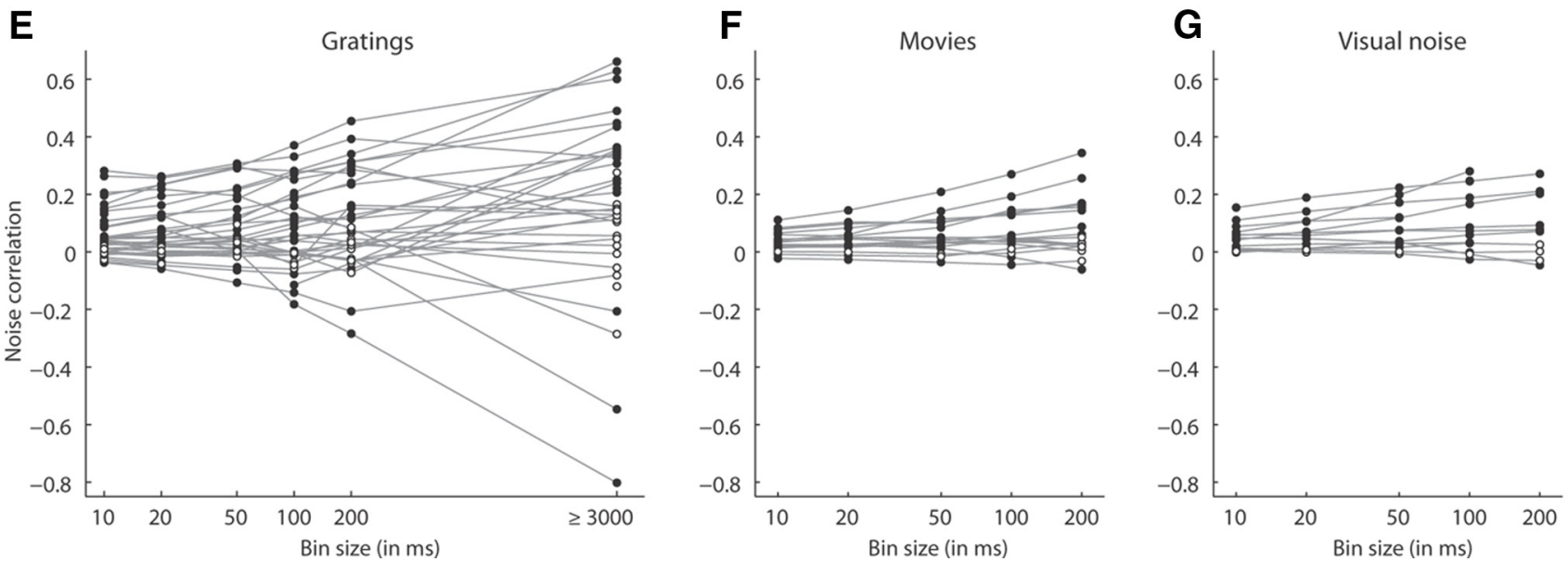

Figure 10. Noise correlations for all stimulus classes. $\boldsymbol{A}$, For two simultaneously recorded neurons (purple and orange traces, respectively; cat0210 P3(4), trial-to-trial fluctuations are plotted for two different presentations (continuous and dashed lines, respectively) of the same $10 \mathrm{~s}$ movie sequence. Mean responses of the same neurons to this movie sequence are shown in Figure $6 C$. Response deviations were determined on bins of $50 \mathrm{~ms}$ and $z$-transformed for each bin with the mean and SD across all 30 stimulus repetitions. $\boldsymbol{B}$, Normalized response deviations from the mean are plotted for both neurons for all presentations of two different movie scenes. Dashed line shows the linear regression line. The noise correlation between these two neurons in response to movies was $0.08(p<0.001)$. C, Distribution of noise correlations on different bin sizes and for different stimulus classes are depicted as box plots (see caption of Fig. $6 E$ for details on box plot representation). The last box shows the distribution of noise correlations measured on complete grating trials (for better visibility, 2 outliers with noise correlations of -0.8 and -0.55 are not shown). The medians of all distributions were significantly larger than zero $(p<0.05$, signed-rank test). $\boldsymbol{D}$, Measured noise correlations are plotted against median of bootstrapped noise correlations for each pair. Lines depict the $95 \%$ confidence interval of the bootstrapped distribution. Dotted gray lines mark equality. Typical number of samples used to determine noise correlations is approximately 14,000 for gratings, 60,000 for movies, and 82,000 for visual noise. These differences might underlie the larger confidence intervals for gratings. $E$, Dependence of noise correlations on bin sizes for each pair of neighboring neurons separately. Gray lines connect noise correlations of each pair. All noise correlations were determined in response to gratings and were measured on complete trials for the largest bin size. Filled dots represent significant noise correlations $(p<0.05) . \boldsymbol{F}, \boldsymbol{G}$, As in $\boldsymbol{E}$ but for noise correlations in response to movies and visual noise, respectively.

dependent similarities in the responses of neighboring neurons, as reflected in their signal correlations, were related to their noise correlations.

\section{Relation between signal and noise correlations depends on stimulus class}

To test for the possible influence of common inputs on signal correlations, we plotted the latter against noise correlations, which were measured on bins of $10 \mathrm{~ms}$ because these are the most robust estimates (see above) and correlate best with signal correlations. Figure $12 \mathrm{~A}$ shows signal correlations on bin sizes of $10 \mathrm{~ms}$ plotted against noise correlations and Figure $12 B$ depicts the strength of this relation for different time scales. The correlation strength between the two measures varied between 0.4 for trial correlations in response to gratings and 0.85 in response to visual noise. For nearby neurons, positive relations between noise and signal correlations or another measure of tuning similarity have also been found in monkey area MT (Zohary et al., 1994; Bair 
et al., 2001). Comparing different time scales, our results show that in response to gratings, signal correlations on larger bins were less well related to noise correlations. We speculate that signal correlations on larger time scales are less dependent on differences between certain RF parameters of the neurons (the data shown in Fig. $8 A$ suggest that, for preferred phase and $\mathrm{RF}$ offset, differences in these parameters might be averaged out for larger bin sizes). These differences may, however, be reflected in the degree of common input and thereby also in the strength of noise correlations of the neurons, which then leads to mismatch between signal and noise correlations. Further analyses will be necessary to substantiate this point. Second, and more importantly, Figure $12 B$ shows that signal and noise correlations were more closely related to each other for gratings and visual noise than for movies. Similar results were observed when noise correlations were measured on larger bin sizes or if we took into account only those pairs for which data to all three stimulus classes were recorded (data not shown). The data shown in Figure $12 \mathrm{~A}$ suggest that the smaller agreement between signal and noise correlations in response to movies is explained by very small noise correlations in pairs that respond similarly to movies (signal correlations $>0.2$ ). Whether this is caused by some form of decorrelation is outside the scope of this study. However, this effect might show specific adaptation of visual cortex to natural stimuli, because low correlations between signal and noise correlations are thought to increase coding capacities (see Discussion).

\section{Firing rates do not account for agreement between noise and signal correlations}

Previous studies have suggested that higher firing rates of neurons could lead to increased noise correlations (de la Rocha et al., 2007; Cohen and Maunsell, 2009). If noise correlations in our data were strongly correlated with firing rates of the neurons and if the same held true for signal correlations, the agreement between signal and noise correlation would be a trivial consequence. In addition, the relation of the correlation measures across stimulus classes might be affected by firing rates. In this section, we will show that firing rates alone cannot explain our results. The distribution of firing rates (Fig. 13A-C) shows that the median firing rates for gratings, movies, and visual noise stimuli were $8.5,4.5$, and $6.0 \mathrm{~Hz}$, respectively. Firing rates in response to gratings were significantly larger than in response to movies ( $p<0.05$, Kruskal-Wallis test across all stimulus classes and Bonferroni correction for multiple comparisons), whereas visual noise evoked firing rates that had magnitudes in between the other two stimulus classes and were not significantly different from them.

For the comparison with signal and noise correlations, the firing rate of a pair was calculated from the firing rate of the less
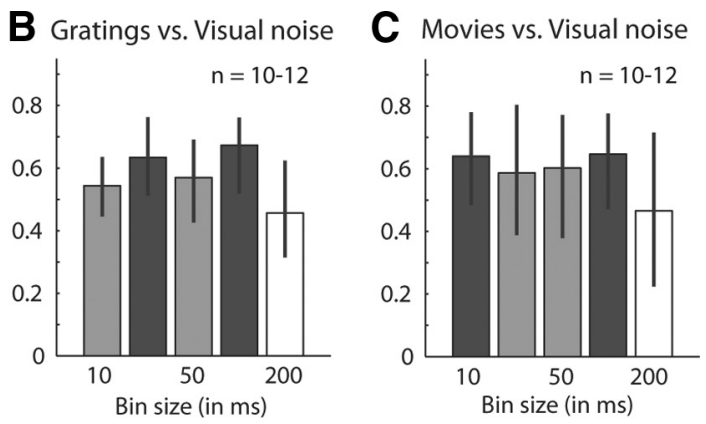

Figure 11. Correlation strengths between noise correlations of different stimulus classes. $\boldsymbol{A}$, Strength of correlation between val based on the botstrapped noise correlations (see Materials and Methods): $n$ is the number of pairs, which differs slightly
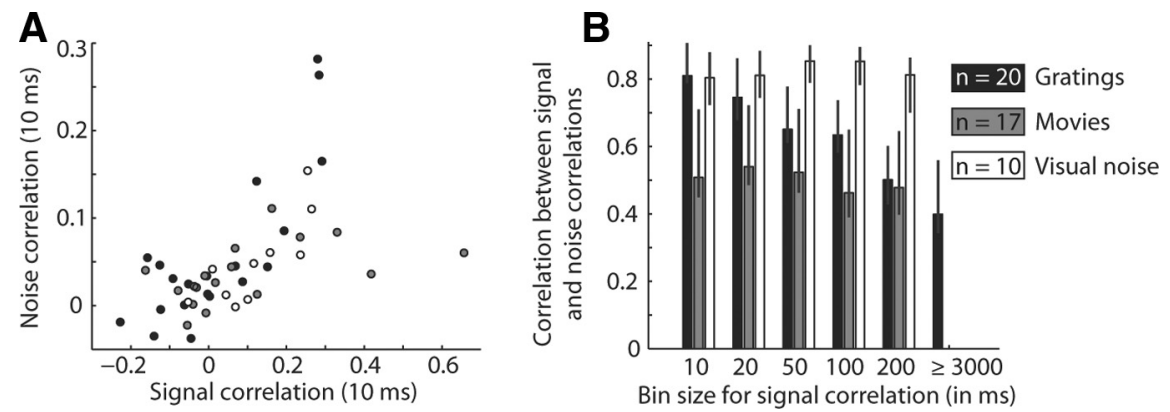

Figure 12. Relation between noise and signal correlations on different time scales. $\boldsymbol{A}$, Noise and signal correlations both (see Materials and Methods). The number of pairs used for each stimulus class is given by $n$. All correlations were significant ( $p<$ 0.05) except movies on bin sizes of $100 \mathrm{~ms}$ and for grating on complete trials.

active neuron in the pair averaged across all stimuli of a class to which the neuron responded reliably. We considered the minimum rate in the pair rather than the commonly used geometric mean rate, because simulations showed that spike count correlations depend more on the minimum response of two neurons than on their mean rate (Fig. $2 D$ in Cohen and Kohn, 2011). The results did not change qualitatively when we used the geometric mean instead of minimum rates. Figure $13 D-F$ shows that signal correlations were positively and significantly correlated with firing rates only in response to gratings and when measured on complete trials ( $p<0.05$, permutation test). For movies and visual noise stimuli, correlations were negative and not significant. The strengths of noise correlations had a positive and significant relationship to firing rates only in response to gratings on bin sizes of $10 \mathrm{~ms}$ (Fig. 13G-I). The relationship in all other cases was nonsignificant $(p>0.05)$.

We then investigated whether firing rates can explain the observed agreements between the correlation measures by determining semipartial correlations. The semipartial correlation between a predictor variable $X$ and a response variable $Y$ expresses the unique contribution of $X$ to the total variance of $Y$ by removing the contribution of another predictor variable, $Z$. Technically, one correlates the residuals from the linear regression of $X$ and $Z$, which removes the effect of $Z$, with $Y$. In our analysis, $X$ and $Y$ are distributions of signal or noise correlations, whereas $Z$ is the distribution of firing rates. We first consider the 
A
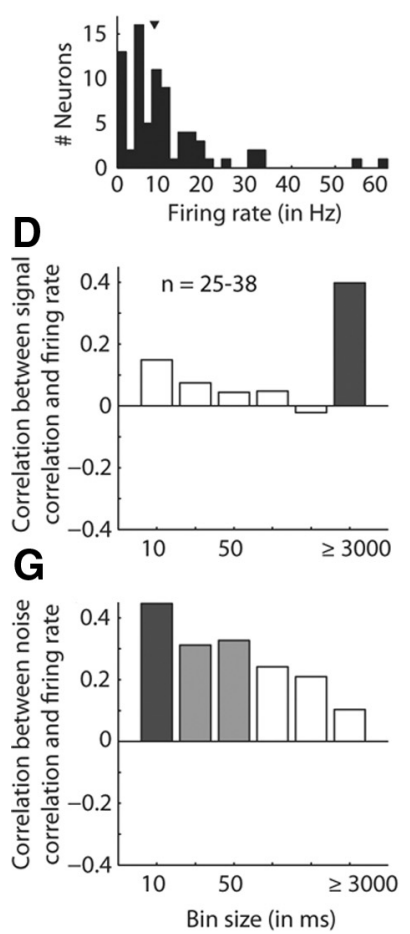

B

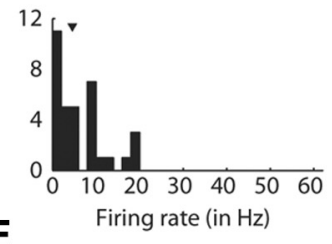

E
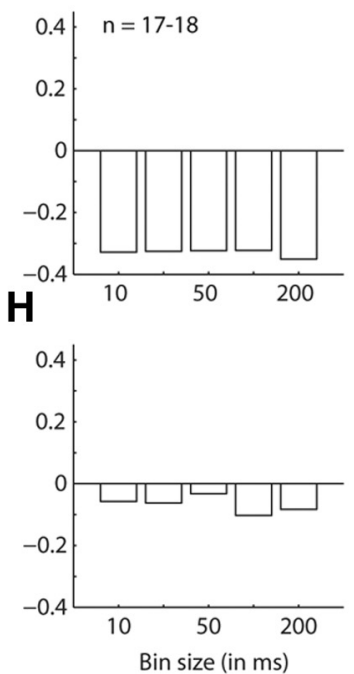

C
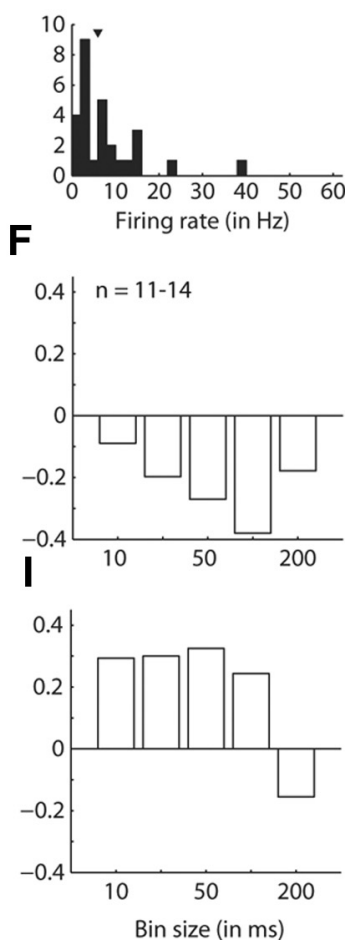

Figure 13. Distribution of firing rates and their relation to signal or noise correlations for gratings $(A, D, G)$, movies $(B, E, H)$, and visual noise $(\boldsymbol{C}, \boldsymbol{F}, \boldsymbol{I})$. $\boldsymbol{A}$, Distribution of firing rates that were averaged across all grating stimuli. $B, C$, same as in $\boldsymbol{A}$ but in response to movies and visual noise, respectively. $\boldsymbol{D}$, Correlation between minimum firing rates in response to gratings and the signal correlations for gratings measured on bins of 10,20,50,100, and $200 \mathrm{~ms}$ and on complete trials; $n$ is the number of pairs, which differs somewhat across bin sizes. Significance levels of correlations are marked by the shade of the bars (dark gray: $p<0.05$, light gray: $p<0.1$, white: $p>0.1) . \boldsymbol{E}, \boldsymbol{F}$, Same as in $\boldsymbol{D}$ but for responses to movies and visual noise stimuli, respectively. $\boldsymbol{G}-\boldsymbol{I}$, Same as in $\boldsymbol{D}-\boldsymbol{F}$ but for noise instead of signal correlations. Number of pairs is the same as for signal correlations.

variance of signal correlations for one stimulus class that can be explained by signal correlations for another stimulus class (Fig. 9). On average, $13.8 \%$ (and at most $36.9 \%$ ) of this variance could be accounted for by firing rates (when comparing the squared correlation coefficient with the squared semipartial correlation coefficient). The strength of correlation between signal correlations of any two stimulus classes was still significant when the explanatory effect of firing rates was accounted for $(p<0.05$, permutation test for semipartial correlation). In the case of noise correlations (Fig. 11), firing rates on average accounted for $5.9 \%$ (maximally for $39.7 \%$ ) of the variance that could be explained by noise correlations of another stimulus class. In most cases, significant relationships between noise correlations of two stimulus classes (Fig. 11) remained significant after the effect of firing rates was accounted for (the only exceptions to that occurred for noise correlations in response to movies and visual noise). Finally, we investigated whether the relation between signal and noise correlations of the neuron pairs (Fig. 12B) could be explained by their firing rates. In all cases in which this relation was significant, maximally $20.9 \%$ of the variance in signal correlations that could be explained by noise correlations, or vice versa, was explained by the minimum firing rates of the pairs (significance levels stayed very similar). Overall, these results indicate that the relationship between the various correlation measures of neighboring neurons cannot be explained by their firing rate alone.

\section{Dependence of response differences on cortical layer}

Neurons situated in different cortical layers play qualitatively different roles in information processing and transmission, so we $5 / 6(p<0.05$, Bonferroni correction; Fig. 14B). Differences between preferences for all other tuning parameters and between tuning widths were very similar between neighboring neurons of all cortical layers.

In agreement with these findings, signal correlations were very similar for all cortical layers, regardless of bin size and stimulus class. For noise correlations, we also found no significant differences between layers. Overall, our results show that the functional neighborhood relationship between neurons in V1 remains fairly similar throughout all cortical layers. The relatively small dataset, however, means that these observations are provisional.

\section{Discussion}

\section{Summary}

In this study, we recorded responses from two to three neighboring neurons in cat visual cortex simultaneously to quantify the differences and similarities between their activity patterns for three different stimulus classes. We found that preferred direction, preferred orientation, and orientation tuning width were more clustered than expected if they were randomly distributed across the cortex. However, preferred phase, direction selectivity, relative modulation, and spatial frequency preference and tuning width show no such clustering. Our results generally agree well with DeAngelis et al. (1999), who measured differences between spatiotemporal RFs of neighboring simple cells in cat area 17, although we included complex cells in our analyses. However, we did not find spatial frequency to be clustered more than expected, which is consistent with other studies on neighboring neurons 

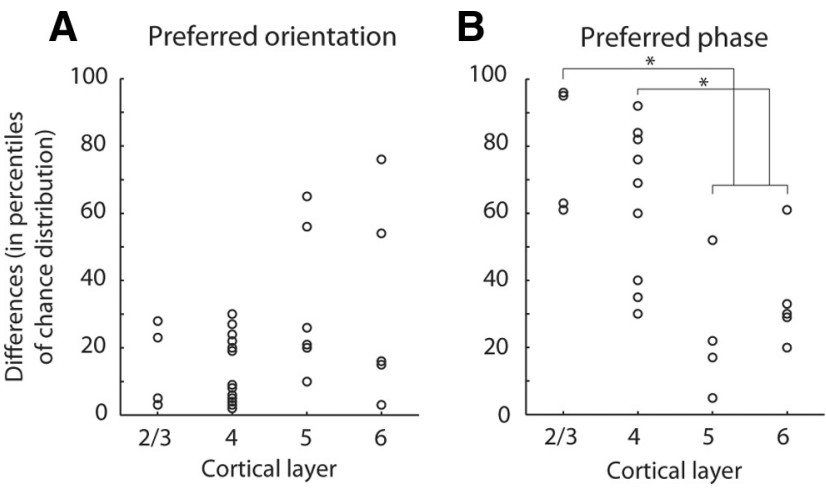

Figure 14. Dependence of response differences between neighboring neurons on the cortical layer. $A$, Differences between preferred orientations of neighboring neurons plotted against the cortical layer of the pair. Differences were expressed as percentiles of the chance distribution (i.e., the differences in preferred orientations between randomly paired neurons). Each circle represents one pair of neighboring neurons. $\boldsymbol{B}$, Same as in $\boldsymbol{A}$ but for differences in preferred phases of neighboring neurons. ${ }^{*}$ Significant differences between layer $2 / 3$, layer 4 , and layers $5 / 6$ (pooled together); $p<0.05$ for Kruskal-Wallis test and accounting for multiple comparisons (see Results).

(including simple and complex cells) in cat area 17 (Tolhurst and Thompson, 1982; Molotchnikoff et al., 2007).

By investigating the temporal patterns in responses of neighboring neurons to movies, visual noise, and gratings, we found that their stimulus-dependent responses, called "signals," showed only small correlations on short time scales (10-200 ms). The strengths of these signal correlations could change with bin size. Although they were similar across all stimulus classes, signal correlations were only weakly related to differences between the neurons' tuning curves. We then measured the correlation between their stimulus-independent responses, called "noise," revealed in the trial-to-trial fluctuations of their responses, because the strength of this correlation is thought to reflect the degree of shared inputs (Shadlen and Newsome, 1998; Bair et al., 2001; Kohn and Smith, 2005). We found small noise correlations and only small differences between stimulus classes. Noise correlations were highly related to signal correlations in response to gratings or visual noise, but less so in response to movies.

\section{Limitations of analyses}

Cohen and Kohn (2011) identified four factors that could bias estimates of noise correlations (and, to some degree, of signal correlations): response strengths, the time period for counting spikes (i.e., bin size), spike sorting, and fluctuations in internal states. We checked for the first two biases by showing that firing rates alone cannot explain our results concerning signal and noise correlations and by considering various time scales for noise and signal correlations, including temporal resolutions of 10 and 20 $\mathrm{ms}$. This range corresponds to the duration of observed membrane time constants and is thus the timescale at which correlated activity most strongly affects the responses of downstream neurons (for review, see Salinas and Sejnowski, 2001). Faulty spike sorting can lead to an overestimation of correlations when spikes of multiple cells are not distinguished, as well as to an underestimation when too many spikes are discarded. We minimized both types of error by achieving a high signal-to-noise ratio in our recordings and by careful screening and identification of all spikes. Slow variations in brain states are generally very hard to assess. We kept the physiological state as constant as possible, as indicated by the vital signs, including the EEG. However, fluctuations on long time scales (several trials) seem to have a minor influence on noise correlations (Bair et al., 2001). Signal correlations could be overestimated if by chance all trials for one stimulus fell into a state of low or high firing rates. It is impossible to exclude such a scenario, but randomization of the stimulus sequence during presentation circumvents this problem in the best possible way.

A further difficulty in measuring signal correlations is that they are completely dependent on the stimulus set. We attempted a meaningful measure of signal correlation by choosing gratings of preferred and nonpreferred values and sampled more finely if online indications were that tuning curves were very narrow. For the visual noise, and especially for movie stimuli, no such strategy exists and the stimulus space is even larger. Signal correlation still appeared to us to be the best measure for tuning similarities because it is independent of any assumptions about the RF structure. However, its limitations must be kept in mind when interpreting our results.

\section{Is coding optimized to natural stimuli?}

A common observation is that noise correlations are largest for neurons with similar tuning properties (for review, see Cohen and Kohn, 2011). Several theoretical studies have indicated that such a correlation structure is highly detrimental for population coding, because responses are harder to decode under these conditions and therefore carry less information about the external stimulus (Abbott and Dayan, 1999; Sompolinsky et al., 2001; Averbeck et al., 2006). Our results on neighboring neurons showed a smaller dependence of noise correlation on signal correlation in response to natural movies, indicative of a more efficient coding. Consistent with this, Vinje and Gallant (2002) found higher information transmission rates for movies than for gratings. To substantiate this apparent adaptation of the brain for efficient coding of natural stimuli, it will be necessary to discover whether and how noise correlations depend on individual stimuli. Stimulus dependence was observed in monkey V1 (Kohn and Smith, 2005) and might largely affect information coding (Averbeck et al., 2006).

The theory of sparse coding argues that natural scenes activate a minimal number of neurons at each point of time (Olshausen and Field, 2004). The significance of this theory comes from its ability to explain the RF structure of simple cells and, more recently, of complex cells, as well as the degree of clustering of RF parameters such as preferred orientation, spatial frequency and phase (Hyvärinen and Hoyer, 2001). Low signal correlations and rare synchronous activity of neighboring neurons are consistent with this idea of sparse coding. However, signal correlations were similarly small for all stimulus classes we considered, not just movies. Furthermore, the lifetime sparseness (a measure of how selectively neurons respond to stimuli) of neurons in awake monkey V1 was similar in response to natural vision and gratings (Vinje and Gallant, 2000). In this sense, there is no sign of a specific adaptation to natural stimuli. However, theoretical predictions as to what the values of sparseness should be in response to artificial stimuli versus natural stimuli do not yet exist.

\section{Information processing in heterogeneous networks}

One possible advantage of cortical maps is that stimulus features can be better estimated when a downstream neuron pools across neurons with similar tuning properties (Parker and Newsome, 1998; Mazurek and Shadlen, 2002). Indeed, neurons in cat V1 are more selective to orientation, which shows a high degree of clustering, than to spatial frequency (Webster and De Valois, 1985). The heterogeneity that we and others have observed, therefore, 
raises a number of questions: how can information be coded and processed, does the functional heterogeneity have any advantages, and what does the underlying circuit look like?

Because of the extensive overlap of their dendritic trees, neighboring neurons are expected to share a large amount of their inputs (Douglas et al., 1995). However, unsynchronized inputs to a neuron, or a balance between excitatory and inhibitory inputs, will not drive the membrane to threshold. Only synchronized excitatory inputs or synchronized inhibitory withdrawal reflected in fast fluctuations in the membrane potential will lead to spikes (Lampl et al., 1999; Hasenstaub et al., 2005; Banitt et al., 2007). Consistently, dual intracellular recordings in cat area 17 show that, whereas the membrane potentials of nearby cells are highly correlated (Yu and Ferster, 2010), only a small fraction of these fluctuations, presumably high-frequency components ( $\mathrm{Ha}-$ senstaub et al., 2005), play a decisive role in triggering spikes. This results in weaker and narrower spike-spike (or noise) correlations (Lampl et al., 1999). Therefore, noise correlations do not reflect the full degree of shared input (de la Rocha et al., 2007; Renart et al., 2010; Cohen and Kohn, 2011), but only the relevant common input that leads to spikes in both neurons. Because our results show a fairly strong relation between noise and signal correlations, it is only this relevant fraction of common input that primarily determines the similarity of the stimulus driven responses of neighboring neurons. This could explain the functional heterogeneity despite shared input.

Pooling across neurons with mixed preferences for one stimulus parameter helps to establish invariance to the parameter in downstream neurons, as observed for the phase invariance in complex cells. However, other nonclustered tuning parameters, such as direction selectivity, still play a crucial role in higher visual areas (Gizzi et al., 1990). In that case, connections to downstream neurons might be highly specific and arise only from neurons that have the same direction selectivity. Some hint of such specificity comes from rodent studies (Yoshimura et al., 2005; Hofer et al., 2011; Ko et al., 2011), but the evidence is weak. In contrast, experimental and theoretical studies suggest that functional heterogeneity in local populations and in downstream inputs does not prohibit high selectivity in postsynaptic neurons (Jia et al., 2010; Hansel and van Vreeswijk, 2012). Pooling across differently tuned neurons is also less likely to amplify noise, which is shared to higher degrees between neurons with similar tuning properties. Finally, a diversity of inputs may be a great advantage for coding the multitude of contexts that a given neuron encounters during the processing of natural scenes.

\section{References}

Abbott LF, Dayan P (1999) The effect of correlated variability on the accuracy of a population code. Neural Comput 11:91-101. CrossRef Medline

Atiya AF (1992) Recognition of multiunit neural signals. IEEE Trans Biomed Eng 39:723-729. CrossRef Medline

Averbeck BB, Latham PE, Pouget A (2006) Neural correlations, population coding and computation. Nat Rev Neurosci 7:358-366. CrossRef Medline

Bair W, Zohary E, Newsome WT (2001) Correlated firing in macaque visual area MT: time scales and relationship to behavior. J Neurosci 21:16761697. Medline

Baker GE, Thompson ID, Krug K, Smyth D, Tolhurst DJ (1998) Spatialfrequency tuning and geniculocortical projections in the visual cortex (areas 17 and 18) of the pigmented ferret. Eur J Neurosci 10:2657-2668. CrossRef Medline

Banitt Y, Martin KA, Segev I (2007) A biologically realistic model of contrast invariant orientation tuning by thalamocortical synaptic depression. J Neurosci 27:10230-10239. CrossRef Medline

Berens P (2009) CircStat: a MATLAB toolbox for circular statistics. Journal of Statistical Software 31:1-21.
Carandini M, Demb JB, Mante V, Tolhurst DJ, Dan Y, Olshausen BA, Gallant JL, Rust NC (2005) Do we know what the early visual system does? J Neurosci 25:10577-10597. CrossRef Medline

Cavanaugh JR, Bair W, Movshon JA (2002) Nature and interaction of signals from the receptive field center and surround in macaque V1 neurons. J Neurophysiol 88:2530-2546. CrossRef Medline

Chklovskii DB, Koulakov AA (2004) Maps in the brain: what can we learn from them? Annu Rev Neurosci 27:369-392. CrossRef Medline

Cohen MR, Kohn A (2011) Measuring and interpreting neuronal correlations. Nat Neurosci 14:811-819. CrossRef Medline

Cohen MR, Maunsell JH (2009) Attention improves performance primarily by reducing interneuronal correlations. Nat Neurosci 12:1594-1600. CrossRef Medline

David SV, Vinje WE, Gallant JL (2004) Natural stimulus statistics alter the receptive field structure of v1 neurons. J Neurosci 24:6991-7006. CrossRef Medline

de la Rocha J, Doiron B, Shea-Brown E, Josić K, Reyes A (2007) Correlation between neural spike trains increases with firing rate. Nature 448:802806. CrossRef Medline

DeAngelis GC, Ghose GM, Ohzawa I, Freeman RD (1999) Functional micro-organization of primary visual cortex: receptive field analysis of nearby neurons. J Neurosci 19:4046-4064. Medline

Douglas RJ, Koch C, Mahowald M, Martin KA, Suarez HH (1995) Recurrent excitation in neocortical circuits. Science 269:981-985. CrossRef Medline

Field DJ (1987) Relations between the statistics of natural images and the response properties of cortical cells. J Opt Soc Am A 4:2379-2394. CrossRef Medline

Gawne TJ, Kjaer TW, Hertz JA, Richmond BJ (1996) Adjacent visual cortical complex cells share about $20 \%$ of their stimulus-related information. Cereb Cortex 6:482-489. CrossRef Medline

Gizzi MS, Katz E, Schumer RA, Movshon JA (1990) Selectivity for orientation and direction of motion of single neurons in cat striate and extrastriate visual cortex. J Neurophysiol 63:1529-1543. Medline

Hansel D, van Vreeswijk C (2012) The mechanism of orientation selectivity in primary visual cortex without a functional map. J Neurosci 32:40494064. CrossRef Medline

Hasenstaub A, Shu Y, Haider B, Kraushaar U, Duque A, McCormick DA (2005) Inhibitory postsynaptic potentials carry synchronized frequency information in active cortical networks. Neuron 47:423-435. CrossRef Medline

Hofer SB, Ko H, Pichler B, Vogelstein J, Ros H, Zeng H, Lein E, Lesica NA, Mrsic-Flogel TD (2011) Differential connectivity and response dynamics of excitatory and inhibitory neurons in visual cortex. Nat Neurosci 14:1045-1052. CrossRef Medline

Hubel DH, Wiesel TN (1962) Receptive fields, binocular interaction and functional architecture in the cat's visual cortex. J Physiol 160:106-154. Medline

Hubel DH, Wiesel TN (1963) Shape and arrangement of columns in cat's striate cortex. J Physiol 165:559-568. Medline

Hubel DH, Wiesel TN (1968) Receptive fields and functional architecture of monkey striate cortex. J Physiol 195:215-243. Medline

Hyvärinen A, Hoyer PO (2001) A two-layer sparse coding model learns simple and complex cell receptive fields and topography from natural images. Vis Res 41:2413-2423. CrossRef Medline

Jia H, Rochefort NL, Chen X, Konnerth A (2010) Dendritic organization of sensory input to cortical neurons in vivo. Nature 464:1307-1312. CrossRef Medline

Ko H, Hofer SB, Pichler B, Buchanan KA, Sjöström PJ, Mrsic-Flogel TD (2011) Functional specificity of local synaptic connections in neocortical networks. Nature 473:87-91. CrossRef Medline

Kohn A, Smith MA (2005) Stimulus dependence of neuronal correlation in primary visual cortex of the macaque. J Neurosci 25:3661-3673. CrossRef Medline

Lampl I, Reichova I, Ferster D (1999) Synchronous membrane potential fluctuations in neurons of the cat visual cortex. Neuron 22:361-374. CrossRef Medline

Mazurek ME, Shadlen MN (2002) Limits to the temporal fidelity of cortical spike rate signals. Nat Neurosci 5:463-471. CrossRef Medline

Mitchell JF, Sundberg KA, Reynolds JH (2009) Spatial attention decorrelates intrinsic activity fluctuations in macaque area V4. Neuron 63:879888. CrossRef Medline 
Molotchnikoff S, Gillet PC, Shumikhina S, Bouchard M (2007) Spatial frequency characteristics of nearby neurons in cats' visual cortex. Neurosci Lett 418:242-247. CrossRef Medline

Mountcastle VB (1957) Modality and topographic properties of single neurons of cat's somatic sensory cortex. J Neurophysiol 20:408-434. Medline

Nover H, Anderson CH, DeAngelis GC (2005) A logarithmic, scale-invariant representation of speed in macaque middle temporal area accounts for speed discrimination performance. J Neurosci 25:10049-10060. CrossRef Medline

Olshausen BA, Field DJ (2004) Sparse coding of sensory inputs. Curr Opin Neurobiol 14:481-487. CrossRef Medline

Parker AJ, Newsome WT (1998) Sense and the single neuron: probing the physiology of perception. Annu Rev Neurosci 21:227-277. CrossRef Medline

Pinter RP, Nabet B (1992) Nonlinear vision: determination of neural fields, function, and networks. Boca Raton, CRC.

Quiroga RQ, Nadasdy Z, Ben-Shaul Y (2004) Unsupervised spike detection and sorting with wavelets and superparamagnetic clustering. Neural Comput 16:1661-1687. CrossRef Medline

Reich DS, Mechler F, Victor JD (2001) Independent and redundant information in nearby cortical neurons. Science 294:2566-2568. CrossRef Medline

Reid RC, Soodak RE, Shapley RM (1987) Linear mechanisms of directional selectivity in simple cells of cat striate cortex. Proc Natl Acad Sci U S A 84:8740-8744. CrossRef Medline

Renart A, de la Rocha J, Bartho P, Hollender L, Parga N, Reyes A, Harris KD (2010) The asynchronous state in cortical circuits. Science 327:587-590. CrossRef Medline

Salinas E, Sejnowski TJ (2001) Correlated neuronal activity and the flow of neural information. Nat Rev Neurosci 2:539-550. CrossRef Medline

Schwartz O, Pillow JW, Rust NC, Simoncelli EP (2006) Spike-triggered neural characterization. J Vis 6:484-507. CrossRef Medline

Shadlen MN, Newsome WT (1998) The variable discharge of cortical neurons: implications for connectivity, computation, and information coding. J Neurosci 18:3870-3896. Medline

Shadlen MN, Britten KH, Newsome WT, Movshon JA (1996) A computational analysis of the relationship between neuronal and behavioral responses to visual motion. J Neurosci 16:1486-1510. Medline

Smith MA, Kohn A (2008) Spatial and temporal scales of neuronal correla- tion in primary visual cortex. J Neurosci 28:12591-12603. CrossRef Medline

Sompolinsky H, Yoon H, Kang K, Shamir M (2001) Population coding in neuronal systems with correlated noise. Phys Rev E Stat Nonlin Soft Matter Phys 64:051904. CrossRef Medline

Swindale NV (1998) Orientation tuning curves: empirical description and estimation of parameters. Biological cybernetics 78:45-56. CrossRef Medline

Tolhurst DJ, Thompson ID (1982) Organization of neurones preferring similar spatial frequencies in cat striate cortex. Exp Brain Res 48:217227. Medline

van Hateren JH, Ruderman DL (1998) Independent component analysis of natural image sequences yields spatio-temporal filters similar to simple cells in primary visual cortex. Proc Biol Sci 265:2315-2320. CrossRef Medline

Vinje WE, Gallant JL (2000) Sparse coding and decorrelation in primary visual cortex during natural vision. Science 287:1273-1276. CrossRef Medline

Vinje WE, Gallant JL (2002) Natural stimulation of the nonclassical receptive field increases information transmission efficiency in V1. J Neurosci 22:2904-2915. Medline

Webster MA, De Valois RL (1985) Relationship between spatial-frequency and orientation tuning of striate-cortex cells. J Opt Soc Am A 2: 1124-1132. CrossRef Medline

Weliky M, Fiser J, Hunt RH, Wagner DN (2003) Coding of natural scenes in primary visual cortex. Neuron 37:703-718. CrossRef Medline

Yen SC, Baker J, Gray CM (2007) Heterogeneity in the responses of adjacent neurons to natural stimuli in cat striate cortex. J Neurophysiol 97:13261341. CrossRef Medline

Yoshimura Y, Dantzker JL, Callaway EM (2005) Excitatory cortical neurons form fine-scale functional networks. Nature 433:868-873. CrossRef Medline

Yu J, Ferster D (2010) Membrane potential synchrony in primary visual cortex during sensory stimulation. Neuron 68:1187-1201. CrossRef Medline

Zohary E, Shadlen MN, Newsome WT (1994) Correlated neuronal discharge rate and its implications for psychophysical performance. Nature 370:140-143. CrossRef Medline 